\title{
P-AREA ACID/CAUSTIC BASIN GROUNDWATER MONITORING REPORT (U)
}

\section{SECOND QUARTER 1995}

\section{Publication Date: September 1995}

Authorized Derivative Classifier and Reviewing Official:

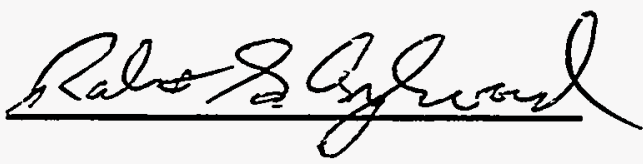

UNCLASSIFIED

Does Not Contain Unclassified Controlled Nuclear Information

Westinghouse Savannah River Company

Savannah River Site

Aiken, SC 29808

Prepared for the U.S. Department of Energy under Control Contract No. DE-AC09-89SR18035 


\section{DISCLAMMER}

This report was prepared as an account of work sponsored by an agency of the United States Government. Neither the United States Government nor any agency thereof, nor any of their employees, makes any warranty, express or implied, or assumes any legal liability or -responsibility for the accuracy, completeness, or usefiulness of any information, apparatus, product, or process disclosed, or represents that its use would not infringe privately owned rights. Reference herein to any specific commercial product, process, or service by trade name, trademark, manufacturer, or otherwise does not necessarily constitute or imply its endorsement, recommendation, or favoring by the United States Government ar any agency thereof. The views and opinions of authors expressed herein do not necessarily state or reflect those of the United States Government or any agency thereof.

This report has been reproduced directly from the best available copy.

Available to DOE and DOE contractors from the Office of Scientific and Technical Information, P.O. Box 62, Oak Ridge, TN 37831; prices available from (615) 576-8401.

Available to the public from the National Technical-Information Service, U.S. Department of Commerce, 5285 Port Royal Road, Springfield, VA 22161. 


\section{P-AREA ACID/CAUSTIC BASIN GROUNDWATER MONITORING \\ REPORT(U)}

\section{SECOND QUARTER 1995}

\section{Publication Date: September 1995}

Key Words

aluminum

iron

manganese

radium-228

Westinghouse Savannah River Company

Savannah River Site

Aiken, SC 29808

Prepared for the U.S. Department of Energy under Control Contract No. DE-AC09-89SR18035

During second quarter 1995, groundwater from the six PAC monitoring wells at the P- 
WSRC-TR-95-0143-2

Unclassified

THIS PAGE LEFT BLANK INTENTIONALLY.

1 


\section{Abstract}

During second quarter 1995, groundwater from the six PAC monitoring wells at the PArea Acid/Caustic Basin was analyzed for herbicides/pesticides, indicator parameters, metals, nitrate, radionuclide indicators, and other constituents. Monitoring results that exceeded the final Primary Drinking Water Standards (PDWS) or Savannah River Site (SRS) flagging criteria such as the SRS turbidity standard during the quarter are discussed in this report.

During second quarter 1995, no constituents exceeded the final PDWS. Aluminum exceeded its SRS Flag 2 criterion in four of the six PAC wells. Iron and manganese exceeded Flag 2 criteria in three wells (PAC 2, 5, and 6). Radium-228 exceeded Level 2 Flagging Criteria in one well (PAC 2); however this was an estimated value because quantitation in the sample did not meet specifications.

Groundwater flow direction and rate in the water table beneath the P-Area Acid/Caustic Basin were similar to past quarters. 
THIS PAGE LEFT BLANK INTENTIONALLY. 


\section{Contents}

Page

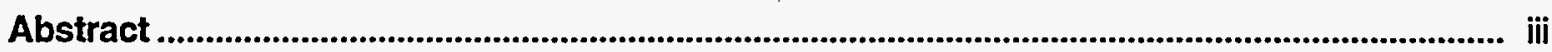

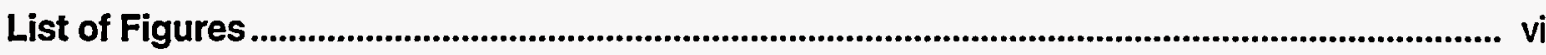

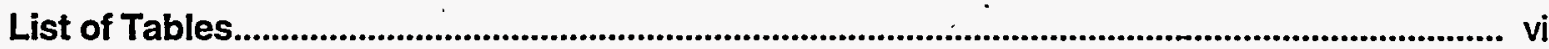

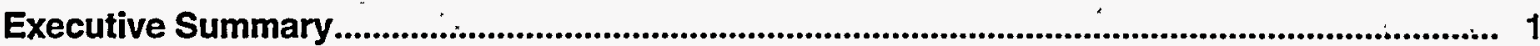

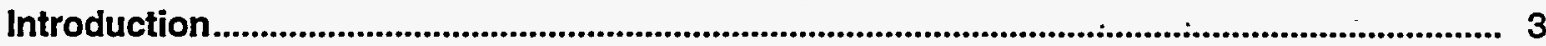

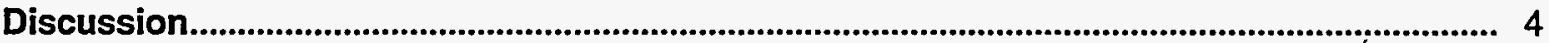

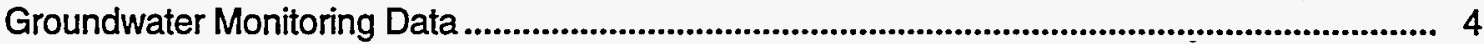

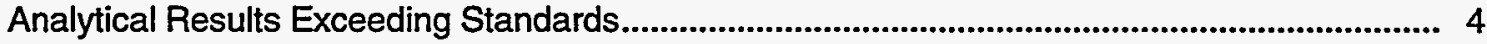

Turbidity Results Exceeding Standards ......................................................................................... 5

Water Elevations, Flow Directions, and Flow Rates ..................................................................... 5

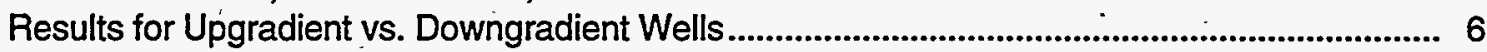

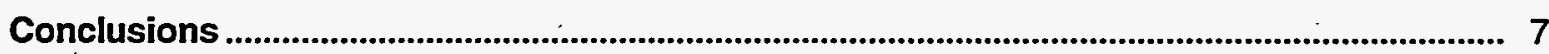

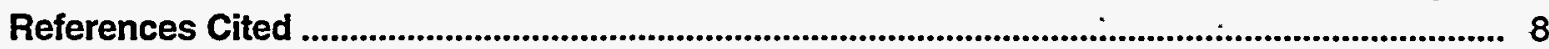

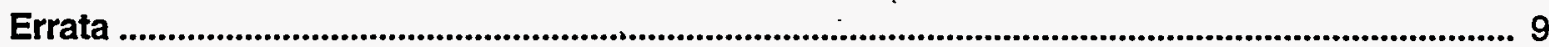

Appendix A_Final Primary Drinking Water Standards ..................................................... A-1

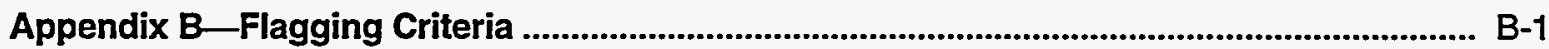

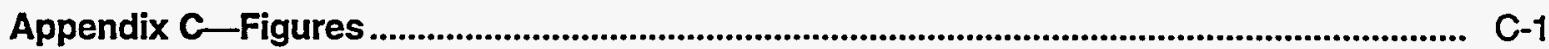

Appendix D—Groundwater Monitoring Results Tables ............................................... D-1

Appendix E-Data Quality/Usability Assessment ................................................................... E-1 


\section{List of Figures}

Page

1. Location of the P-Area Acid/Caustic Basin at the Savannah River Site

C-3

2. Location of Groundwater Monitoring Wells at the P-Area Acid/Caustic Basin

C-4

3. Piezometric Surface Map of the Water Table at the P-Area Acid/Caustic

Basin.

\section{List of Tables}

Page

1. Maximum Results for Constituents Exceeding Final Primary Drinking Water Standards....

D-6

2. Maximum Results for Constituents Exceeding Other Flag 2 Criteria or the SRS Turbidity Standard

3. Groundwater Monitoring Results for Individual Wells 


\section{Executive Summary}

The PAC monitoring wells at the P-Area Acid/Caustic Basin are sampled quarterly as part of the Savannah River Site (SRS) Groundwater Monitoring Program and to comply with the terms of a consent decree signed May 26, 1988, by the U.S. District Court (District of South Carolina, Aiken Division).

During second quarter 1995, groundwater from the PAC wells received analyses for herbicides/ pesticides, indicator parameters, metals, nitrate, radionuclide indicators, and other constituents. Monitoring results that exceeded the final Primary Drinking Water Standards (PDWS) or the SRS flagging criteria or turbidity standard are discussed in this report.

During second quarter 1995, no constituents exceeded the final PDWS in wells at the P-Area Acid/Caustic Basin. Aluminum exceeded its SRS Flag 2 criterion in four of the six PAC wells with values ranging from $124 \mu \mathrm{g} / \mathrm{L}$ to $288 \mu \mathrm{g} / \mathrm{L}$. Iron and manganese exceeded Flag 2 criteria in wells PAC 2, 5, and 6 . The values for iron ranged from 761 $\mu \mathrm{g} / \mathrm{L}$ to $5440 \mu \mathrm{g} / \mathrm{L}$ and the manganese values ranged from $61.6 \mu \mathrm{g} / \mathrm{L}$ to $306 \mu \mathrm{g} / \mathrm{L}$.

Groundwater flow direction to the north-northwest at a rate of $2433 \mathrm{ft} / y e a r$ in the water table beneath the P-Area Acid/Caustic Basin was similar to past quarters. 
THIS PAGE LEFT BLANK INTENTIONALLY. 


\section{Introduction}

The P-Area Acid/Caustic Basin is located east of P-Area and Road F at the Savannah River Site (SRS) (Figure 1, Appendix C) on a slope that leads to a tributary of Par Pond. The following description outlines important events in the history of the P-Area Acid/Caustic Basin.

- The basin, constructed in the early 1950s, is an unlined earthen pit that received . dilute sulfuric acid and sodium hydroxide solutions and other wastes from several areas within SRS. The basin provided an area for the mixing and neutralization of the dilute solutions before their discharge to nearby streams (Heffner and Exploration Resources, 1991).

- The P-Area Acid/Caustic Basin remained in service until new neutralization facilities became operational in 1982 (Heffner and Exploration Resources, 1991).

- Groundwater monitoring wells PAC 1,2, 3, and 4 (Figure.2, Appendix C) were installed at the P-Area Acid/Caustic Basin between November 1983 and July 1984 (EPD/EMS, 1994).

- Under the terms of a consent decree signed May 26, 1988, by the U.S. District Court (Civil Action 1:85-2583-6, District of South Carolina, Aiken Division), the basin became subject to requirements of Subtitle $C$ of the Resource Conservation and Recovery Act (RCRA), the South Carolina Hazardous Waste Management Regulations (SCHWMR), and associated regulations on June 1, 1988.

- The basin's monitoring wells were reevaluated during the summer of 1988 to ensure compliance with SCHWMR. As part of this compliance effort, wells PAC 5 and 6 (Figure 2, Appendix C) were installed at the P-Area Acid/Caustic Basin during fall 1988 (EPD/EMS, 1994).

- The revised Groundwater Quality Assessment Plan (WSRC, 1991), submitted to the South Carolina Department of Health and Environmental Control (SCDHEC) on April 30, 1991, indicated that the monitoring well network at the P-Area Acid/Caustic Basin is sufficient to detect effects of the basin on the groundwater.

- During July through September 1993, with SCDHEC's permission to proceed at risk, SRS stabilized the $\mathrm{H}$ - and P-Area Acid/Caustic Basins as proposed in the Interim Status Closure Plan for the F-, H-, K-, and P-Area Acid/Caustic Basins (Revision 3, February 5,1992$)$. The basins were dewatered and filled with compacted clay-rich soil, and a vegetative cover of winter- and drought-hardy grass was established.

Each quarter, the Environmental Protection Department/Environmental Monitoring Section (EPD/EMS) samples the monitoring wells at the P-Area Acid/Caustic Basin as part of the SRS Groundwater Monitoring Program. The Environmental Restoration Department provides a quarterly report describing the monitoring results to SCDHEC in compliance with SCHWMR. 


\section{Discussion}

\section{Groundwater Monitoring Data}

The EPD/EMS groundwater sampling procedure (EPD/EMS, 1992) requires evacuation of a minimum of two well volumes and stabilization of $\mathrm{pH}$, specific conductance, and turbidity prior to sample collection. Stability is established when a minimum of three successive measurements, taken within a given time period, are within a specified tolerance range. If a well pumps dry before two well volumes are purged or before stabilization is achieved, it must be revisited within 24 hours for the data to be considered the result of a single sampling event. On the second visit within 24 hours, samples are taken without purging or measuring stability; thus, these samples may not be representative of the groundwater quality.

During second quarter 1995, samples from the six monitoring wells at the P-Area Acid/Caustic Basin were analyzed for herbicides/pesticides, indicator parameters, metals, nitrate, radionuclide indicators, and other constituents. This report describes results that equaled or exceeded the Safe Drinking Water Act final Primary Drinking Water Standards (PDWS) or drinking water screening levels, as éstablished by the U.S. Environmental Protection Agency (EPA) (Appendix A); the South Carolina final PDWS for lead (Appendix A); SRS flagging criteria that are based on final and proposed PDWS, Secondary Drinking Water Standards, and method detection limits (Appendix B); or the SRS turbidity standard. Constituent levels that equaled or exceeded the final PDWS, screening levels, or other Flag 2 criteria are described as exceeding standards, above standards, or as elevated.

The final PDWS for individual analytes presented in Appendix A may not always match the SRS flagging criteria presented in Appendix B. The final PDWS generally are used in this compliance report as guidelines to meet regulatory requirements; the flagging criteria are used by EPD/EMS to identify relative levels of constituents in the groundwater and as guides for scheduling groundwater monitoring.

\section{Analytical Results Exceeding Standards}

No analytes exceeded the final PDWS (Appendix A) during second quarter 1995; see Table 1 (Appendix D). Constituents that exceeded other Flag 2 criteria (Appendix B) during second quarter 1995 are summarized in Table 2 (Appendix D). Aluminum exceeded the Flag 2 criterion in four PAC wells, with a maximum concentration of $288 \mu$ $\mathrm{g} / \mathrm{L}$ in well PAC 1. Iron and manganese exceeded Flag 2 criteria in wells $P A C 2,5$, and 6. Iron had a maximum concentration of $5440 \mu \mathrm{g} / \mathrm{L}$ in well PAC 2 , and manganese had a maximum concentration of $306 \mu \mathrm{g} / \mathrm{L}$ in well PAC 5. Radium-228 also exceeded Level 2 Flagging Criteria in well PAC 222 with a reported value of $63.7 \mathrm{~J} \mathrm{pCi/L}$. The "J" modifier indicates that the reported value is an estimated quantity because quantitation in the sample or in associated quality contral samples did not meet specifications.

Table 3 (Appendix D) presents all of the results for individual wells and indicates the analytical laboratories that conducted the analyses, the dilution factors used in the analyses, and the analyses that received modifiers (which help identify laboratory accuracy and precision) or that exceeded the EPA-approved holding times during second quarter 1995. Constituent results in Table 3 that appear to equal the final PDWS but are not marked in the ST column (exceeded final PDWS or screening level) are below the 
final PDWS in the database. Database results, the results that are compared to the final PDWS, are entered with more significant digits than the results given in this report. Apparent discrepancies are the result of the rounding of reported results.

Table 3 also lists the number of well volumes purged from each well during second quarter 1995 and provides a statement that describes incomplete or unsuccessful sampling events. Wells PAC 5 and 6 failed to yield enough water to meet the purging and stabilization criteria; thus, these wells may not have produced representative groundwater samples.

Appendix $D$ provides definitions of the abbreviations and the modifiers used in the results tables as well as descriptions of holding times, data rounding, and data qualification practices.

Appendix $\mathrm{E}$ provides a general assessment of the quality and usability of the data provided by EPD/EMS.

\section{Turbidity Results Exceeding Standards}

Turbidity results, in nephelometric turbidity units (NTU), of less than 5 NTU meet the EPA's general standard for acceptability of groundwater samples (EPA, 1986). That standard, however, is considered unrealistic for monitoring wells at SRS. Gass (1989) has documented turbidity measurements ranging up to 5,000 NTU from properly designed wells screened in poorly productive formations. During the 1989 RCRA Compliance Evaluation Inspection, officials from EPA Region IV indicated that the SRS turbidity standard of 50 NTU is conservative. These officials also agreed that water-table wells in this area often screen nonaquifer formations, rendering development of these. wells more difficult due to the low yield and high proportion of mobile fines typical of these formations (Bergren and Bennett, 1989).

Turbidity results from wells PAC 1, 5 , and 6 were between the EPA standard of 5 NTU and the SRS standard of 50 NTU. Well PAC 5 exhibited the highest turbidity of any PAC well this quarter with 15.4 NTU.

\section{Water Elevations, Flow Directions, and Flow Rates}

Water-table elevations and the groundwater flow direction beneath the P-Area Acid/Caustic Basin are shown in Figure 3 (Appendix C). Water-table elevations indicate that the groundwater flow direction is north-northwest (using universal transverse Mercator coordinates).

The groundwater flow rate in the water table (Aquifer Zone $\|_{1} B_{2}$ ) beneath the P-Area Acid/Caustic Basin is estimated using the following equation:

$$
\text { Flow }(\mathrm{ft} / \text { day })=\frac{\text { Hydraulic Conductivity }(\mathrm{ft} / \text { day })}{\text { Porosity (unitless) }} \times \frac{d h(\mathrm{ft})}{d l(\mathrm{ft})}
$$

A hydraulic conductivity constant of $10 \mathrm{ft} /$ day (Geraghty \& Miller, Inc., 1990) is a conservative estimate (i.e., the actual hydraulic conductivity should be somewhat less than $10 \mathrm{ft} /$ day). 
The effective porosity is estimated at 20 percent (Killian et al., 1987); $d$ is the difference in head, and $d /$ is the length of the flow path to the nearest $10 \mathrm{ft}$. Flow rate estimates vary depending on the vertical gradient between wells, the size of the area under consideration, and the number of data points. The flow path is measured along the map; as much as possible, the path is drawn the same each quarter. Because of these variables, the estimation of flow rate must be considered accurate to an order of magnitude only.

Flow rate estimates are calculated as follows: flow rate per day is calculated to two significant figures using the above equation. This value is then multiplied by 365 and rounded to two significant figures for the flow rate per year.

Using the above equation with $d h=20 \mathrm{ft}$ and $d l=150 \mathrm{ft}$, the flow rate estimate for groundwater in the water table beneath the P-Area Acid/Caustic Basin (Figure 3, Appendix C) is as follows:

$$
\frac{10}{0.2} \times \frac{20}{150}=6.6 \mathrm{ft} / \mathrm{day}^{\prime}
$$

$6.6 \mathrm{ft} /$ day $\times 365$ days $\approx 2433 \mathrm{ft} /$ year

\section{Results for Upgradient vs. Downgradient·Wells}

Wells PAC 1 and 4 are the upgradient wells at the P-Area Acid/Caustic Basin. Wells $P A C 2,3,5$, and 6 are downgradient. During second quarter 1995, no constituents exceeded the final PDWS in the upgradient or downgradient wells. Aluminum exceeded its SRS Flag 2 criterion in both the upgradient and two of the downgradient wells. Iron and manganese were elevated in downgradient wells PAC 2, 5, and 6. 


\section{Conclusions}

During second quarter 1995, no analytes exceeded the final PDWS in any wells at the P-Area Acid/Caustic Basin. Aluminum exceeded its SRS Flag 2 criterion in both of the upgradient wells and two of the downgradient wells. Iron and manganese each exceeded the Flag 2 criterion in downgradient wells PAC 2, 5, and 6. Generally, constituents found in downgradient wells but not in upgradient wells at a waste management unit are considered products of the waste management unit.

Water-table elevations at the P-Area Acid/Caustic Basin indicate that groundwater flow direction was north-northwest (UTM coordinates) at a rate of approximately $2433 \mathrm{ft} /$ year. Groundwater flow direction in this area can be accurately defined because the groundwater gradient is large and follows the surface topography. The revised Groundwater Quality Assessment Plan (WSRC, 1991) for the P-Area Acid/Caustic Basin indicates that the monitoring well network at the basin is sufficient to detect effects of past basin operations on groundwater quality. 


\section{References Cited}

Bergren, C. L., and C. B. Bennett, 1989. Assessment of SRS Groundwater Monitoring Wells Impacted by Turbidity, WSRC-RP-89-891. Westinghouse Savannah River Company, Aiken, SC.

EPA (U.S. Environmental Protection Agency), 1986. RCRA Ground Water Monitoring Technical Enforcement Guidance Dócument, OSWER-9950.1. Washington, DC.

EPD/EMS (Environmental Protection Department/Environmental Monitoring Section), 1992. Hydrogeologic Data Collection Procedures and Specifications: Sampling Groundwater Monitoring Wells, Manual 3Q5, Chapter 14, Revision 0. Environmental Protection Department, Environmental Monitoring Section, Savannah River Site, Aiken, SC.

EPD/EMS (Environmental Protection Department/Environmental Monitoring Section), 1994. Environmental Protection Department's Well Inventory (through the second quarter of 1994), ESH-EMS-940518. Westinghouse Savannah River Company, Savannah River Site, Aiken, SC.

Gass, T. E., 1989. Monitoring Wells in Non-Aquifer Formations. Water Well Journal 43(2):2729.

Geraghty \& Miller, Inc., 1990. Evaluation of Integrated Waste Facility Closure Capping on Ground-Water Flow and Solute Transport in General Separations Area, Savannah River Site: Flow Model and Particle-Tracking Analysis, Final Report. Prepared by Geraghty \& Miller Modeling Group for Westinghouse Savannah River Company, Waste Management Technology, Savannah River Site, Aiken, SC.

Heffner, J.D., and Exploration Resources, Inc., 1991. Technical Summary of Groundwater Quality Protection Program at the Savannah River Site (1952-1986), Volume - Site Geohydrology and Waste Sites, DPSP-88-1002. Westinghouse Savannah River Company, Aiken, SC.

Killian, T. H., N. L. Kolb, P.Corbo, and I. W. Marine, 1987. F-Area Seepage Basins, DPST-85-704. Savannah River Laboratory, E.I. duPont de Nemours \& Company, Aiken,SC.

WSRC (Westinghouse Savannah River Company), 1991. F-, H-, K-, and P-Area Acid/Caustic Basins Groundwater Quality Assessment Plan, WSRC-TR-91-178, Revision 1.0. Westinghouse Savannah River Company, Aiken, SC.

WSRC, 1995. Closure Plan for the F-, H-, K-, and P-Area Acid/Caustic Basins (U), WSRCRP-94-1259, Revision 6. Westinghouse Savannah River Company, Aiken, SC. 


\section{Errata}

In tables with four quarters of data, some values for earlier quarters may differ from values for those same quarters presented in earlier reports because some reanalyses may have been performed by the laboratories after the reports were printed.

Second quarter 1995:

- No errata have been reported. 
THIS PAGE LEFT BLANK INTENTIONALLY. 


\section{Appendix A}

Final Primary Drinking Water Standards 
. THIS PAGE LEFT BLANK INTENTIONALLY. 
Final Primary Drinking Water Standards

\begin{tabular}{|c|c|c|c|c|}
\hline Analyte & Unit & Level & Status & Source \\
\hline Alachlor & $\mu \mathrm{g} / \mathrm{L}$ & 2 & Final & EPA, 1993 \\
\hline Aldicarb ${ }^{a}$ & $\mu g / L$ & 3 & Final & EPA, 1993 \\
\hline Aldicarb sulfone $e^{a}$ & $\mu g / L$ & 2 & Final & EPA, 1993 \\
\hline Aldicarb sulfoxide ${ }^{a}$ & $\mu g / L$ & 4 & Final & EPA, 1993 \\
\hline Antimony & $\mu \mathrm{g} / \mathrm{L}$ & 6 & Final & EPA, 1993 \\
\hline Arsenic & $\mu g / L$ & 50 & Final & EPA, 1993 \\
\hline Asbestos & Fibers/L & $7,000,000$ & Final & EPA, 1993 \\
\hline Atrazine & $\mu g / L \quad$. & 3 & Final & EPA, 1993 \\
\hline Barium & $\mu g / L$ & 2,000 & Final & EPA, 1993 \\
\hline Benzene & $\mu \mathrm{g} / \mathrm{L}$ & 5 & Final & EPA, 1993 \\
\hline Benzo[a]pyrene & $\mu g / L$ & 0.2 & Final & EPA, 1993 \\
\hline Beryllium & $\mu g / L$ & 4 & Final & EPA, 1993 \\
\hline Bis(2-ethylhexyl) phthalate & $\mu g / L$ & 6 & Final & EPA, 1993 \\
\hline Bromodichloromethane & $\mu \mathrm{g} / \mathrm{L}$ & 100 & Final & EPA, 1993 \\
\hline Bromoform & $\mu \mathrm{g} / \mathrm{L}$ & 100 & Final & EPA, 1993 \\
\hline 2-sec-Butyl-4,6-dinitrophenol & $\mu g / L$ & 7 & Final & EPA, 1993 \\
\hline Cadmium & $\mu g / L$ & 5 & Final & EPA, 1993 \\
\hline Carbofuran & $\mu g / L$ & 40 & Final & EPA, 1993 \\
\hline Carbon tetrachloride & $\mu g / L$ & 5 & Final & EPA, 1993 \\
\hline Chlordane & $\mu g / L$ & 2 & Final & EPA, 1993 \\
\hline Chlorobenzene & $\mu g / L$ & 100 & Final & EPA, 1993 \\
\hline Chloroethene (Vinyl chloride) & $\mu \mathrm{g} / \mathrm{L}$ & 2 & Final & EPA, 1993 \\
\hline Chloroform & $\mu g / L$ & 100 & Final & EPA, 1993 \\
\hline Chromium & $\mu g / L$ & 100 & Final & EPA, 1993 \\
\hline Copper & $\mu \mathrm{g} / \mathrm{L}$ & 1,300 & Final & EPA, 1993 \\
\hline Cyanide & $\mu g / L$ & 200 & Final & EPA, 1993 \\
\hline Dalapon ${ }^{\mathrm{a}}$ & $\mu g / L$ & 200 & Final & EPA, 1993 \\
\hline Dibromochloromethane & $\mu g / L$ & 100 & Final & EPA, 1993 \\
\hline 1,2-Dibromo-3-chloropropane & $\mu g / L$ & $0: 2$ & Final & EPA, 1993 \\
\hline 1,2-Dibromoethane & $\mu g / L$ & 0.05 & Final & EPA, 1993 \\
\hline 1,2-Dichlorobenzene & $\mu g / L$ & 600 & Final & EPA, 1993 \\
\hline 1,4-Dichlorobenzene & $\mu \mathrm{g} / \mathrm{L}$ & 75 & Final & EPA, 1993 \\
\hline 1,2-Dichloroethane & $\mu g / L$ & 5 & Final & EPA, 1993 \\
\hline 1,1-Dichloroethylene & $\mu g / L$ & 7 & Final & EPA, 1993 \\
\hline 1,2-Dichloroethylene & $\mu g / L$ & 50 & Final & EPA, 1993 \\
\hline cis-1,2-Dichloroethylene & $\mu g / L$ & 70 & Final & EPA, 1993 \\
\hline trans-1,2-Dichloroethylene & $\mu g / L$ & 100 & Final & EPA, 1993 \\
\hline Dichloromethane (Methylene chloride) & $\mu g / L$ & 5 & Final & EPA, 1993 \\
\hline 2,4-Dichlorophenoxyacetic acid & $\mu g / L$ & 70 & Final & EPA, 1993 \\
\hline 1,2-Dichloropropane & $\mu g / L$ & 5 & Final & EPA, 1993 \\
\hline Di(2-ethylhexyl) adipate ${ }^{a}$ & $\mu g / L$ & 400 & Final & EPA, 1993 \\
\hline Diquat dibromide $^{a}$ & $\mu g / L$ & 20 & Final & EPA, 1993 \\
\hline Endothalla & $\mu g / L$ & 100 & Final & EPA, 1993 \\
\hline Endrin & $\mu \mathrm{g} / \mathrm{L}$ & 2 & Final & EPA, 1993 \\
\hline Ethylbenzene & $\mu \mathrm{g} / \mathrm{L}$ & 700 & Final & EPA, 1993 \\
\hline Fluoride & $\mu g / L$ & 4,000 & Final & EPA, 1993 \\
\hline Glyphosate $^{a}$ & $\mu g / L$ & 700 & Final & EPA, 1993 \\
\hline Gross alphab & $\mathrm{pCi} / \mathrm{L}$ & $1.5 E+01$ & Final & EPA, 1993 \\
\hline Heptachlor & $\mu \mathrm{g} / \mathrm{L}$ & 0.4 & Final & EPA, 1993 \\
\hline
\end{tabular}


Heptachlor epoxide

Hexachlorobenzene

Hexachlorocyclopentadiene

Lead

Lindane

Mercury

Methoxychlor

Nickel

Nitrate as nitrogen

Nitrate-nitrite as nitrogen

Nitrite as nitrogen

Nonvolatile beta

Oxamyla

PCB 1016

PCB 1221

PCB 1232

PCB 1242

PCB 1248

PCB 1254

PCB 1260

PCB 1262

Pentachlorophenol

Piclorama

Selenium

Simazinea

Strontium-89/90c

Strontium-90

Styrene

2,3,7,8-TCDD

Tetrachloroethylene

Thallium

Toluene

Toxaphene

2,4,5-TP (Silvex)

1,2,4-Trichlorobenzene

1,1,1-Trichloroethane

1,1,2-Trichloroethane

Trichloroethylene

Tritium

Xylenes

\begin{tabular}{|c|c|c|c|}
\hline$\mu g / L$ & 0.2 & & Final \\
\hline$\mu g / L$ & 1 & & Final \\
\hline$\mu g / L$. & 50 & & Final \\
\hline$\mu g / L$ & $50^{\prime}$ & & Final \\
\hline$\mu g / L$ & 0.2 & & Final \\
\hline$\mu \mathrm{g} / \mathrm{L}$ & 2 & & Final \\
\hline$\mu g / L$ & 40 & & Final \\
\hline$\mu g / L$ & 100 & & Final \\
\hline$\mu g / L$ & 10,000 & & Final \\
\hline$\mu \mathrm{g} / \mathrm{L}$ & 10,000 & & Final \\
\hline$\mu g / L$ & 1,000 & . & Final \\
\hline $\mathrm{pCi} / \mathrm{L}$ & $5 E+01$ & & Interim Final \\
\hline$\mu g / L$ & 200 & & Final \\
\hline$\mu g / L$ & 0.5 & & Final \\
\hline$\mu g / L$ & 0.5 & & Final \\
\hline$\mu g / L$ & 0.5 & & Final \\
\hline$\mu g / L$ & 0.5 & & Final \\
\hline$\mu g / L$ & 0.5 & & Final \\
\hline$\mu \mathrm{g} / \mathrm{L}$ & 0.5 & & Final \\
\hline$\mu g / L$ & 0.5 & & Final \\
\hline$\mu g / L$ & 0.5 & & Final \\
\hline$\mu g / L$ & 1 & & Final \\
\hline$\mu g / L$ & 500 & & Final \\
\hline$\mu g / L$ & 50 & & Final \\
\hline$\mu g / L$ & 4 & & Final \\
\hline pCi/L & $8 E+00$ & & Final \\
\hline $\mathrm{pCi} / \mathrm{L}$ & $8 E+00$ & & Final \\
\hline$\mu g / L$ & 100 & & Final \\
\hline$\mu g / L$ & 0.00003 & & Final \\
\hline$\mu g / L$ & 5 & & Final \\
\hline$\mu g / L$ & 2 & & Final \\
\hline$\mu g / L$ & 1,000 & & Final \\
\hline$\mu g / L$ & 3 & & Final \\
\hline$\mu g / L$ & 50 & & Final \\
\hline$\mu g / L$ & 70 & & Final \\
\hline$\mu g / L$ & 200 & & -Final \\
\hline$\mu g / L$ & 5 & $\cdot$ & Final \\
\hline$\mu g / L$ & 5 & & Final \\
\hline $\begin{array}{l}\mathrm{pCi} / \mathrm{mL} \\
\mu \mathrm{g} / \mathrm{L}\end{array}$ & $\begin{array}{l}2 E+01 \\
10,000\end{array}$ & & $\begin{array}{l}\text { Final } \\
\text { Final }\end{array}$ \\
\hline
\end{tabular}

EPA, 1993

EPA, 1993

EPA; 1993

SCDHEC, 1981

EPA, 1993

EPA, 1993

EPA, 1993

EPA, 1993

EPA, 1993

EPA, 1993

EPA, 1993

EPA, 1977

EPA, 1993

EPA, 1993

EPA, 1993

EPA, 1993.

$\because$ EPA, 1993

EPA; 1993

EPA, 1993

EPA, 1993

EPA, 1993

EPA, 1993

EPA, 1993

EPA, 1993

EPA, 1993

EPA, 1993

EPA, 1993

EPA, 1993

EPA, 1993

EPA, 1993

EPA, 1993

EPA, 1993

EPA, 1993

EPA, 1993

EPA, 1993

EPA, 1993

EPA, 1993

EPA, 1993

EPA, 1993

EPA, 1993

Note: Final PDWS were assigned to alachlor, aldicarb, aldicarb sulfone, aldicarb sulfoxide, atrazine, carbofuran, dalapon, di(2-ethylhexyl) adipate, diquat dibromide, endothall, glyphosate, oxamyl, picloram, and simazine in the SRS Groundwater Monitoring Program for the first time beginning first quarter 1994.

a At present, EMS does not perform this analysis because the constituent is not in the current contract.

The standard given is for gross alpha including radium-226 but excluding radion and uranium.

c For double radionuclide analyses where each separate radionuclide has its own standard, the more stringent standard is used. 


\section{References}

EPA (U.S. Environmental Protection Agency), 1977. National Interim Primary Drinking Water Regulations, EPA-570/9-76-003. Washington, DC.

EPA (U.S. Environmental Protection Agency), 1993. National Primary Drinking Water Regulations, Code of Federal Regulations, Title 40, Part 141, pp. 592-732. Washington, DC.

SCDHEC (South Carolina Department of Health and Environmental Control), 1981. State Primary Drinking Water Regulations, R.61-58.5. Columbia, SC. 
THIS PAGE LEFT BLANK INTENTIONALLY. 


\title{
Appendix B
}

\author{
Flagging Criteria
}


THIS PAGE LEFT BLANK INTENTIONALLY.' 


\section{Flagging Criteria}

The Savannah River Site Environmental Protection Department/Environmental Monitoring Section (EPD/EMS) flagging criteria are as follows:

- Flag 2 criteria for constituents equal the Safe Drinking Water Act (SDWA) final Primary Drinking Water Standards (PDWS), the SDWA proposed PDWS, or the SDWA Secondary Drinking Water Standards (SDWS). If a constituent does not have a drinking water standard, the Flag 2 criterion equals 10 times the method detection limit (MDL) calculated as the 90th percentile. detection limit obtained recently by one of the primary analytical laboratories.

- Flag 1 criteria for constituents equal one-half of the final PDWS, one-half the proposed PDWS, or one-half the SDWS. If a constituent does not have a drinking water standard, the Flag 1 criterion equals 5 times the MDL calculated as the 90th percentile detection limit obtained recently by one of the primary analytical laboratories.

- Flag 0 criteria are assigned to constituent levels below Flag 1 criteria, constituent levels below the sample detection limits, or constituents having no flagging criteria.

The following parameters are exceptions to the flagging rules:

- EPD/EMS sets flagging criteria for $\mathrm{pH}$ and specific conductance. No flags are set for alkalinity, calcium, carbonate, magnesium, potassium, silica, sodium, total dissolved solids, total phosphates (as P), and total phosphorus. Analyses for these parameters are conducted as part of the biennial comprehensive analyses or by special request.

- Aesthetic parameters such as color, corrosivity, Eh, odor, surfactants, and turbidity are not assigned flagging criteria but are analyzed by special request.

- Common laboratory contaminants and cleaners such as dichloromethane (methylene chloride), ketones, phthalates, and toluene are not assigned flagging criteria unless they have primary drinking water standards. These constituents are analyzed by special request.

\begin{tabular}{|c|c|c|c|c|}
\hline Analyte & Unit & Flag 1 & Flag 2 & Source $^{a}$ \\
\hline Acenaphthene & $\mu g / L$ & 50 & 100 & EPA Method 8270 \\
\hline Acenaphthylene & $\mu g / L$ & 50 & 100 & EPA Method 8270 \\
\hline Acetone & $\mu g / L$ & 500 & 1,000 & EPA Method 8240 \\
\hline Acetonitrile (Methyl cyanide) & $\mu g / L$ & 500 & 1,000 & EPA Method 8240 \\
\hline Acetophenone & $\mu g / L$ & 50 & 100 & EPA Method 8270 \\
\hline 2-Acetylaminofluorene & $\hat{\mu g} / \mathrm{L}$ & 50 & 100 & EPA Method 8270 \\
\hline Acrolein & $\mu g / L$ & $100^{\circ}$ & 200 & EPA Method 8240 \\
\hline Acrylonitrile & $\mu g / L$ & 100 & 200 & EPA Method 8240 \\
\hline Actinium-228 & $\mathrm{pCi} / \mathrm{L}$ & $1.64 E+03$ & $3.27 E+03$ & Proposed PDWS (EPA, 1991) \\
\hline Alachlor & $\mu g / L$ & 1 & 2 & Final PDWS (EPA, 1993a) \\
\hline Aldicarb $b^{b}$ & $\mu g / L$ & 1.5 & 3 & Final PDWS (EPA, 1993a) \\
\hline Aldicarb sulfone ${ }^{b}$ & $\mu g / L$ & 1 & 2 & Final PDWS (EPA, 1993a) \\
\hline Aldicarb sulfoxide $b$ & $\mu g / L$ & 2 & 4 & Final PDWS (EPA, 1993a) \\
\hline Aldrin & $\mu g / L$ & 0.25 & 0.5 & EPA Method 8080 \\
\hline Alkalinity (as $\mathrm{CaCO}_{3}$ ) & & No flag & No flag & Set by EPD/EMS \\
\hline Allyl chloride & $\mu g / L$ & 250 . & 500 & EPA Method 8240 \\
\hline Aluminum & $\mu g / L$ & 25 & 50 & SDWS (EPA, 1993b) \\
\hline Aluminum, dissolved & $\mu g / L$ & 25 & 50 & SDWS.(EPA, 1993b) \\
\hline
\end{tabular}


Aluminum, total recoverable

Americium-241

Americium-243

4-Aminobiphenyl

Ammonia

Ammonia nitrogen

Aniline

Anthracene

Antimony

Antimony, dissolved ...

Antimony, total recoverable

Antimony-125

Aramite

Arsenic

Arsenic, dissolved

Arsenic, total recoverable

Asbestos

Atrazine

Azobénzene

Barium

Barium, dissolved

Barium, total recoverable

Barium-140c

Benzene

alpha-Benzene hexachloride

beta-Benzene hexachloride

delta-Benzene hexachloride

Benzidine

Benzo[a]anthracene

Benzo[b]fluoranthene

Benzo[kffluoranthene

Benzoic acid

Benzo $[g, h$,$] perylene$

Benzo[a]pyrene

1,4-Benzoquinone

Benzyl alcohol

Beryllium

Beryllium, dissolved

Beryllium, total recoverable

Beryllium-7

Bis(2-chloroethoxy) methane

Bis(2-chloroethyl) ether

Bis(2-chloroisopropyl) ether

Bis(chloromethyl) ether

Bis(2-ethylhexyl) phthalate

Bismuth-214

Boron

Boron, dissolved

Boron, total recoverable

Bromide

Bromodichloromethane

Bromoform

Bromomethane (Methyl bromide)

4-Bromophenyl phenyl ether

\begin{tabular}{|c|c|c|}
\hline$\mu \mathrm{g} / \mathrm{L}$ & 25 & 50 \\
\hline $\mathrm{pCi} / \mathrm{L}$ & 3.17E+00 & $6.34 E+00$ \\
\hline $\mathrm{pCi} / \mathrm{L}$ & $3.19 E+00$ & $6.37 E+00$ \\
\hline$\mu \mathrm{g} / \mathrm{L}$ & 50 & 100 \\
\hline$\mu \mathrm{g} / \mathrm{L}$ & 500 & 1,000 \\
\hline$\mu g / L$ & 500 & 1,000 \\
\hline$\mu g / L$ & 50 & 100 \\
\hline$\mu \mathrm{g} / \mathrm{L}$ & 50 & 100 \\
\hline$\mu \mathrm{g} / \mathrm{L}$ & 3 & 6 \\
\hline$\mu g / L$ & 3 & 6 \\
\hline$\mu \mathrm{g} / \mathrm{L}$ & 3 & 6 \\
\hline $\mathrm{pCi} / \mathrm{L}$ & $1.5 E \div 02$ & $3 E+02$ \\
\hline$\mu g / L$ & 50 & 100 \\
\hline$\mu g / L$ & 25 & 50 \\
\hline$\mu g / L$ & 25 & 50 \\
\hline$\mu g / L$ & 25 & 50 \\
\hline Fibers/L & $3,500,000$ & $7,000,000$ \\
\hline$\mu g / L$ & 1.5 & 3 \\
\hline$\mu g / L$ & 50 & 100 \\
\hline$\mu \mathrm{g} / \mathrm{L}$ & 1,000 & 2,000 \\
\hline$\mu \mathrm{g} / \mathrm{L}$ & 1,000 & 2,000 \\
\hline$\mu g / L$ & 1,000 & 2,000 \\
\hline $\mathrm{pCi} / \mathrm{L}$ & $4.5 \mathrm{E}+01$ & $9 E+01$ \\
\hline$\mu \mathrm{g} / \mathrm{L}$ & 2.5 & 5 \\
\hline$\mu \mathrm{g} / \mathrm{L}$ & 0.25 & 0.5 \\
\hline$\mu \mathrm{g} / \mathrm{L}$ & 0.25 & 0.5 \\
\hline$\mu g / L$ & 0.25 & 0.5 \\
\hline$\mu \mathrm{g} / \mathrm{L}$ & 250 & 500 \\
\hline$\mu \mathrm{g} / \mathrm{L}$ & 0.05 & 0.1 \\
\hline$\mu \mathrm{g} / \mathrm{L}$ & 0.1 & 0.2 \\
\hline$\mu \mathrm{g} / \mathrm{L}$ & 0.1 & 0.2 \\
\hline$\mu \mathrm{g} / \mathrm{L}$ & 250 & 500 \\
\hline$\mu \mathrm{g} / \mathrm{L}$ & 50 & 100 \\
\hline$\mu \mathrm{g} / \mathrm{L}$ & 0.1 & 0.2 \\
\hline$\mu \mathrm{g} / \mathrm{L}$ & 50 & 100 \\
\hline$\mu \mathrm{g} / \mathrm{L}$ & 50 & 100 \\
\hline$\mu \mathrm{g} / \mathrm{L}$ & 2 & 4 \\
\hline$\mu g / L$ & 2 & 4 \\
\hline$\mu g / L$ & 2 & 4 \\
\hline $\begin{array}{l}\mathrm{pCi} / \mathrm{L} \\
\mu \mathrm{g} / \mathrm{L}\end{array}$ & $\begin{array}{l}3 E+03 \\
50\end{array}$ & $\begin{array}{l}6 E+03 \\
100\end{array}$ \\
\hline$\mu \mathrm{g} / \mathrm{L}$ & 50. & 100 \\
\hline$\mu \mathrm{g} / \mathrm{L}$ & 50 & 100 \\
\hline$\mu \mathrm{g} / \mathrm{L}$ & 50 & 100 \\
\hline$\mu g / L$ & 3 & 6 \\
\hline $\mathrm{pCi} / \mathrm{L}$ & $9.4 E+03$ & $1.89 E+04$ \\
\hline$\mu g / L$ & 150 & 300 \\
\hline$\mu g / L$ & 150 & 300 \\
\hline$\mu \mathrm{\mu g} / \mathrm{L}$ & 150 & 300 \\
\hline$\mu g / L$ & 5,000 & 10,000 \\
\hline$\mu \mathrm{g} / \mathrm{L}$ & 50 & 100 \\
\hline$\mu g / L$ & 50 & 100 \\
\hline$g / L$ & 5 & 10 \\
\hline a/l & 50 & 100 \\
\hline
\end{tabular}

SDWS (EPA, 1993b)

Proposed PDWS (EPA, 1991)

Proposed PDWS (EPA, 1991)

EPA Method 8270

APHA Method 417B

EPA Method 350.1

EPA Method 8270

EPA Method 8270

Final PDWS (EPA, 1993a)

Final PDWS (EPA, 1993a)

Final PDWS (EPA, 1993a)

Interim Final PDWS (EPA, 1977)

EPA Method 8270

Final PDWS (EPA, 1993a)

Final PDWS (EPA, 1993a)

Final PDWS (EPA, 1993a)

Final PDWS (EPA, 1993a)

Final PDWS (EPA, 1993a)

EPA Method 625

Final PDWS (EPA, 1993a)

Final PDWS (EPA, 1993a)

Final PDWS (EPA, 1993a)

Interim Final PDWS (EPA, 1977)

Final PDWS (EPA, 1993a)

EPA Method 8080

EPA Method 8080

EPA Method 8080

EPA Method 8270

Proposed PDWS (EPA, 1990)

Proposed PDWS (EPA, 1990)

Proposed PDWS (EPA, 1990)

EPA Method 8270

EPA Method 8270

Final PDWS (EPA, 1993a)

EPA Method 8270

EPA Method 8270

Final PDWS (EPA, 1993a)

Final PDWS (EPA, 1993a)

Final PDWS (EPA, 1993a)

Interim Final PDWS (EPA, 1977)

EPA Method 8270

EPA Method 8270

EPA Method 8270

EPA Method 8270

Final PDWS (EPA, 1993a)

Proposed PDWS (EPA, 1991)

EPA Method 6010

EPA Method 6010

EPA Method 6010

EPA Method 300.0

Final PDWS (EPA, 1993a)

Final PDWS (EPA, 1993a)

EPA Method 8240

EPA Method 8270 
Butylbenzyl phthalate

2-sec-Butyl-4,6-dinitrophenol

Cadmium

Cadmium, dissolved

Cadmium, total recoverable

Calcium

Calcium, dissolved

Calcium, total recoverable

Carbofuran

Carbon-14

Carbonate

Carbon disulfide

Carbon tetrachloride

Cerium- $141^{\mathrm{C}}$

Cerium-144

Cesium-134 ${ }^{\mathrm{d}}$

Cesium-137

Chlordane

Chloride

4-Chloroaniline

Chlorobenzene

Chlorobenzilate

4-Chloro-m-cresol

Chloroethane

Chloroethene (Vinyl chloride)

Chloroethyl vinyl ether

2-Chloroethyl vinyl ether

Chloroform

Chloromethane (Methyl chloride)

2-Chloronaphthalene

2-Chlorophenol

4-Chlorophenyl phenyl ether

Chloroprene

Chromium

Chromium, dissolved

Chromium, total recoverable

- Chromium-51c

Chrysene

Cobalt

Cobalt, dissolved

Cobalt, total recoverable

Cobalt-57

Cobalt-58

Cobalt-60

Color

Copper

Copper, dissolved

Copper, total recoverable

Corrosivity

m-Cresol (3-Methylphenol)

o-Cresol (2-Methylphenol)

p-Cresol (4-Methylphenol)

Curium-242

Curium-243

Curium-243/244e

\begin{tabular}{|c|c|c|c|}
\hline & No flag & No flag & Set by EPD/EMS \\
\hline$\mu \mathrm{g} / \mathrm{L}$ & 3.5 & 7 & Final PDWS (EPA, 1993a) \\
\hline$\mu \mathrm{g} / \mathrm{L}$ & 2.5 & 5 & Final PDWS (EPA, 1993a) \\
\hline$\mu \mathrm{g} / \mathrm{L}$ & 2.5 & 5 & Final PDWS (EPA, 1993a) \\
\hline \multirow[t]{4}{*}{$\mu \mathrm{g} / \mathrm{L}$} & 2.5 & 5 & Final PDWS (EPA, 1993a) \\
\hline & No flag & No flag & Set by EPPD/EMS \\
\hline & No flag & No flag & Set by EPD/EMS \\
\hline & No flag & No flag & Set by EPD/EMS \\
\hline$\mu g / L$ & 20 & 40 & Final PDWS (EPA, 1993a) \\
\hline \multirow[t]{2}{*}{$\mathrm{pCi} / \mathrm{L}$} & $1 E+03$ & $2 E+03$ & Interim Final PDWS (EPA, 1977) \\
\hline & No flag & No flag & Set by EPD/EMS \\
\hline$\mu \mathrm{g} / \mathrm{L}$ & 5 & 10 & EPA Method 8240 \\
\hline$\mu g / L$ & 2.5 & 5 & Final PDWS (EPA, 1993a) \\
\hline $\mathrm{pCi} / \mathrm{L}$ & $1.5 E+02$ & $3 E+02$ & Interim Final PDWS (EPA, 1977) \\
\hline $\mathrm{pCi} / \mathrm{L}$ & $1.31 E+02$ & $2.61 E+02$ & Proposed PDWS (EPA, 1991) \\
\hline $\mathrm{pCi} / \mathrm{L}$ & $4.07 E+01$ & $8.13 E+01$ & Proposed PDWS (EPA, 1991) \\
\hline $\mathrm{pCi} / \mathrm{L}$ & $1 E+02$ & $2 \mathrm{E}+02$ & Interim Final PDWS (EPA, 1977) \\
\hline$\mu g / L$ & 1 & 2 & Final PDWS (EPA, 1993a) \\
\hline$\mu \mathrm{g} / \mathrm{L}$ & 125,000 & 250,000 & SDWS (EPA, 1993b) \\
\hline$\mu \mathrm{g} / \mathrm{L}$ & 50 & 100 & EPA Method 8270 \\
\hline$\mu \mathrm{g} / \mathrm{L}$ & 50 & 100 & Final PDWS (EPA, 1993a) \\
\hline$\mu \mathrm{g} / \mathrm{L}$ & 50 & 100 & EPA Method 8270 \\
\hline$\mu \mathrm{g} / \mathrm{L}$ & 50 & 100 & EPA Method 8270 \\
\hline$\mu \mathrm{g} / \mathrm{L}$ & 5 & 10 & EPA Method 8240 \\
\hline$\mu \mathrm{g} / \mathrm{L}$ & 1 & 2 & Final PDWS (EPA, 1993a) \\
\hline$\mu g / L$ & 5 & 10 & EPA Method 8240 \\
\hline$\mu \mathrm{g} / \mathrm{L}$ & 5 & 10 & EPA Method 8240 \\
\hline$\mu \mathrm{g} / \mathrm{L}$ & 50 & 100 & Final PDWS (EPA, 1993a) \\
\hline$\mu \mathrm{g} / \mathrm{L}$ & 5 & 10 & EPA Method 8240 \\
\hline$\mu \mathrm{g} / \mathrm{L}$ & 50 & 100 & EPA Method 8240 \\
\hline$\mu \mathrm{g} / \mathrm{L}$ & 50 & 100 & EPA Method 8270 \\
\hline$\mu \mathrm{g} / \mathrm{L}$ & 50 & 1,00 & EPA Method 8270 \\
\hline$\mu \mathrm{g} / \mathrm{L}$ & 1,000 & 2,000 & EPA Method 8240 \\
\hline$\mu g / L$ & 50 & $100^{\circ}$ & Final PDWS (EPA, 1993a) \\
\hline$\mu g / L$ & 50 & 100 & Final PDWS (EPA, 1 \\
\hline$\mu g / L$ & 50 & 100 & Finàl PDWS (EPA, 1993a) \\
\hline $\mathrm{pCi} / L$ & $3 E+03$ & $6 \mathrm{E}+03$ & Interim Final PDWS (EPA, 1977) \\
\hline$\mu g / L$ & 0.1 & 0.2 & Proposed PDWS (EPA, 1990) \\
\hline$\mu g / L$ & 20 & 40 & EPA Method 6010 \\
\hline$\mu \mathrm{g} / \mathrm{L}$ & 20 & 40 & EPA Method 6010 \\
\hline$\mu g / L$ & 20 & 40 & EPA Method 6010 \\
\hline $\mathrm{pCi} / \mathrm{L}$ & $5 E+02$ & $1 E+03$ & Interim Final PDWS (EPA, 1977) \\
\hline $\mathrm{pCi} / \mathrm{L}$ & $4.5 E+03$ & $9 \mathrm{E}+03$ & Interim Final PDWS (EPA, 1977) \\
\hline $\mathrm{pCi} / \mathrm{L}$ & $\begin{array}{l}5 \mathrm{E}+01 \\
\text { No flag }\end{array}$ & $\begin{array}{l}1 E+02 \\
\text { No flag }\end{array}$ & $\begin{array}{l}\text { Interim Final PDWS (EPA, 1977) } \\
\text { Set by EPD/EMS }\end{array}$ \\
\hline$\mu \mathrm{g} / \mathrm{L}$ & 500 & 1,000 & Final PDWS (SCDHEC, 1981) \\
\hline$\mu \mathrm{g} / \mathrm{L}$ & 500 & 1,000 & Final PDWS (SCDHEC, 1981) \\
\hline$\mu g / L$ & 500 & 1,000 & Final PDWS (SCDHEC, 1981) \\
\hline & No flag & No flag & Set by EPD/EMS \\
\hline$\mu \mathrm{g} / \mathrm{L}$ & 50 & 100 & EPA Method 8270 \\
\hline$\mu \mathrm{g} / \mathrm{L}$ & 50 & 100 & EPA Method 8270 \\
\hline$\mu g / L$ & 50 & 100 & EPA Method 8270 \\
\hline $\mathrm{pCi} / \mathrm{L}$ & $6.65 E+01$ & $1.33 E+02$ & Proposed PDWS. (EPA, 1991) \\
\hline $\mathrm{pCi} / \mathrm{L}$ & 4.15E+00 & $8.3 E+00$ & Proposed PDWS (EPA, 1991) \\
\hline $\mathrm{pCi} / \mathrm{L}$ & $4.15 E+00$ & $8.3 E+00$ & Proposed PDWS (EPA, 1991) \\
\hline
\end{tabular}




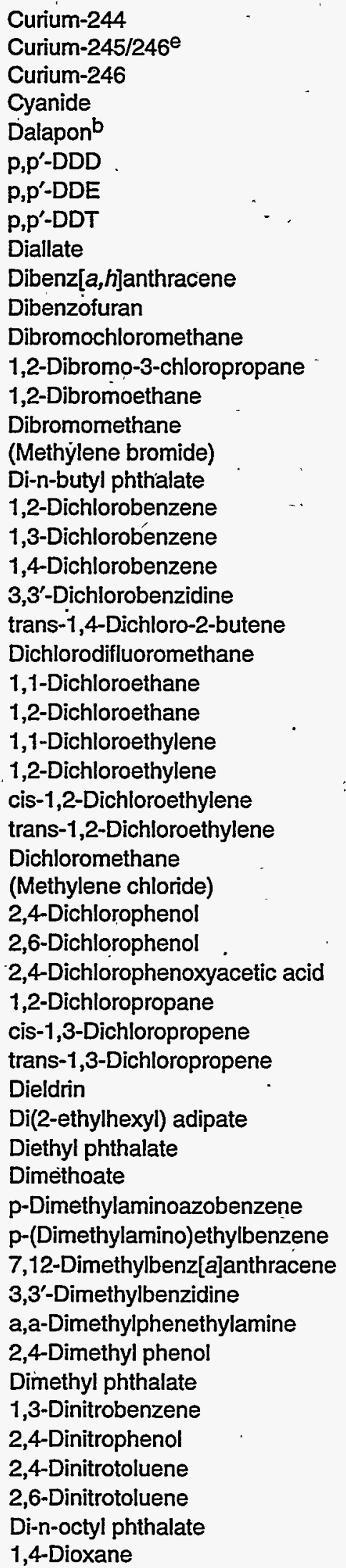

\begin{tabular}{|c|c|c|c|}
\hline $\mathrm{pCi} / \mathrm{L}$ & $4.92 E+00$ & $9.84 \mathrm{E}+00$ & Proposed PDWS (EPA, 1991) \\
\hline $\mathrm{pCi} / \mathrm{L}$ & $3.12 E+00$ & $6.23 E+00$ & Proposed PDWS (EPA, 1991) \\
\hline $\mathrm{pCi} / \mathrm{L}$ & $3.14 E+00$ & $6.27 E+00$ & Proposed PDWS (EPA, 1991) \\
\hline$\mu g / L$ & 100 & 200 & Final PDWS (EPA, 1993a) \\
\hline$\mu g / L$ & 100 & 200 & Final PDWS (EPA, 1993a) \\
\hline$\mu g / L$ & 0.5 & 1. & EPA Method 8080 \\
\hline$\mu \mathrm{g} / \mathrm{L}$ & 0.5 & 1 & EPA Method 8080 \\
\hline$\mu g / L$ & 0.5 & 1 & EPA Method 8080 \\
\hline$\mu \mathrm{g} / \mathrm{L}$ & 50 & 100 & EPA Method 8270 \\
\hline$\mu g / L$ & 0.15 & 0.3 & Proposed PDWS (EPA, 1990) \\
\hline$\mu g / L$ & 50 & 100 & EPA Method 8270 \\
\hline$\mu g / L$ & 50 & 100 & Final PDW'S (EPA, 1993a) \\
\hline$\mu \mathrm{g} / \mathrm{L}$ & 0.1 & 0.2 & Final PDWS (EPA, 1993a) \\
\hline$\mu \mathrm{g} / \mathrm{L}$ & 0.025 & 0.05 & Final PDWS (EPA, 1993a) \\
\hline$\mu g / L$ & 5 & 10 & EPA Method 8240 \\
\hline$\mu g / L$ & $\begin{array}{l}\text { No flag } \\
300\end{array}$ & $\begin{array}{l}\text { No flag } \\
600\end{array}$ & $\begin{array}{l}\text { Set by EPD/EMS } \\
\text { Final PDWS (EPA, 1993a) }\end{array}$ \\
\hline$\mu \mathrm{g} / \mathrm{L}$ & 50 & 100 & EPA Method 8270 \\
\hline$\mu \mathrm{g} / \mathrm{L}$ & 37.5 & 75 & Final PDWS (EPA, 1993a) \\
\hline$\mu \mathrm{g} / \mathrm{L}$ & .50 & 100 & EPA Method 8270 \\
\hline$\mu g / L$ & 150 & 300 & EPA Method 8240 \\
\hline$\mu \mathrm{g} / \mathrm{L}$ & 5 & 10 & EPA Method 8240 \\
\hline$\mu g / L$ & 5 & 10 & EPA Method 8240 \\
\hline$\mu g / L$ & 2.5 & 5 & Final PDWS (EPA, 1993a) \\
\hline$\mu g / L$ & 3.5 & 7 & Final PDWS (EPA, 1993a) \\
\hline$\mu g / L$ & 25 & 50 & Final PDWS (EPA, 1993a) \\
\hline$\mu g / L$ & 35 . & 70 & Final PDWS (EPA, 1993a) \\
\hline$\mu g / L$ & 50 & 100 & Final PDWS (EPA, 1993a) \\
\hline$\mu g / L$ & 2.5 & 5 & Final PDWS (EPA, 1993a) \\
\hline$\mu g / L$ & 50 & 100 & EPA Method 8270 \\
\hline$\mu \mathrm{g} / \mathrm{L}$ & 50 & 100 & EPA Method 8270 \\
\hline$\mu g / L$ & 35 & 7.0 & Final PDWS (EPA, 1993a) \\
\hline$\mu g / L$ & 2.5 & 5 & Final PDWS (EPA, 1993a) \\
\hline$\mu \mathrm{g} / \mathrm{L}$ & 5 & 10 & EPA Method 8240 \\
\hline$\mu \mathrm{g} / \mathrm{L}$ & 5 & 10 & EPA Method 8240 \\
\hline$\mu g / L$ & 2.5 & 5 & EPA Method 8080 \\
\hline$\mu g / L$ & 200 & 400 & Final PDWS (EPA, 1993a) \\
\hline & No flag & No flag. & Set by EPD/EMS \\
\hline$\mu g / L$ & 50 & 100 & EPA Method 8270 \\
\hline$\mu g / L$ & 50 & 100 & EPA Method 8270 \\
\hline$\mu g / L$ & 50 & 100 & EPA Method 8270 \\
\hline$\mu g / L$ & 50 & 100 & EPA Method 8270 \\
\hline$\mu g / L$ & 50 & 100 & EPA Method 8270 \\
\hline$\mu g / L$ & 50 & 100 & EPA Method 8270 \\
\hline$\mu g / L$ & 50 & 100 & EPA Method 8270 \\
\hline & No flag & No flag & Set by EPD/EMS \\
\hline$\mu g / L$ & 50 & 100 & EPA Method 8270 \\
\hline$\mu g / L$ & 250 & 500 & EPA Method 8270 \\
\hline$\mu g / L$ & 50 & 100 & EPA Method 8270 \\
\hline$\mu g / L$ & 50 & 100 & EPA Method 8270 \\
\hline & No flag & No flag & Set by EPD/EMS \\
\hline$\mu g / L$ & 50 & 100 & EPA Method 8270 \\
\hline
\end{tabular}


Diphenylamine

1,2-Diphenylhydrazine

Diquat dibromide ${ }^{b}$

Dissolved organic carbon

Disulfoton

Eh

Endosulfan I

Endosulfan II

Endosulfan sulfate

Endothall ${ }^{b}$

Endrin

Endrin aldehyde

Endrin ketone

Ethylbenzene

Ethyl methacrylate

Ethyl methanesulfonate

Europium-152

Europium-154

Europium-155

Famphur

Fluoranthene

Fluorene

Fluoride

Glyphosate ${ }^{b}$

Gross alpha

Heptachlor

Heptachlor epoxide

Heptachlorodibenzo-p-dioxin

isomers

1,2,3,4,6,7,8-HPCDD

Heptachlorodibenzo-p-furan isomers

1,2,3,4,6,7,8-HPCDF

Hexachlorobenzene

Hexachlorobutadiene

Hexachlorocyclopentadiene

Hexachlorodibenzo-p-dioxin isomers

1,2,3,4,7,8-HXCDD

Hexachlorodibenzo-p-furan isomers

1,2,3,4,7,8-HXCDF

Hexachloroethane

Hexachlorophene

Hexachloropropene

2-Hexanone

Indeno[1,2,3-c, d]pyrene

lodine

lodine-129

lodine-131 $c$

lodomethane (Methyl iodide)

Iron

Iron, dissolved

Iron, total recoverable

Iron-55c

\begin{tabular}{|c|c|c|c|}
\hline$\mu \mathrm{g} / \mathrm{L}$ & 50 & 100 & EPA Method 8270 \\
\hline$\mu \mathrm{g} / \mathrm{L}$ & 50 & 100 & EPA Method 8270 \\
\hline$\mu \mathrm{g} / \mathrm{L}$ & 10 & 20 & Final PDWS (EPA, 1993a) \\
\hline$\mu g / L$ & 5,000 & 10,000 & EPA Method 9060 \\
\hline$\mu g / L$ & 50 & 100 & EPA Method 8270 \\
\hline & No flag & No flag & Set by EPD/EMS \\
\hline$\mu \mathrm{g} / \mathrm{L}$ & 0.5 & 1 & EPA Method 8080 \\
\hline$\mu \mathrm{g} / \mathrm{L}$ & 0.5 & 1 & EPA Method 8080 \\
\hline$\mu \mathrm{g} / \mathrm{L}$ & 0.5 & 1 & EPA Method 8080 \\
\hline$\mu \mathrm{g} / \mathrm{L}$ & 50 & 100 & Final PDWS (EPA, 1993a) \\
\hline$\mu \mathrm{g} / \mathrm{L}$ & 1 & .2 & Fịnal PDWS (EPA, 1993a) \\
\hline$\mu \mathrm{g} / \mathrm{L}$ & 0.5 & 1 & EPA Method 8080 \\
\hline & No flag & No flag & Set by EPD/EMS \\
\hline$\mu \mathrm{g} / \mathrm{L}$ & 350 & 700 & Final PDWS (EPA, 1993a) \\
\hline$\mu g / L$. & 50 & 100 & EPA Method 8270 \\
\hline$\mu g / L$ & 50 & 100 & EPA Method 8270 \\
\hline $\mathrm{pCi} / \mathrm{L}$ & $3 E+01$ & $6 E+01^{\circ}$ & Interim Final PDWS (EPA, 1977) \\
\hline $\mathrm{pCi} / \mathrm{L}$ & $1 E+02$ & $2 E+02$ & Interim Final PDWS (EPA, 1977) \\
\hline $\mathrm{pCi} / \mathrm{L}$ & $3 E+02$ & $6 E+02$ & Interim Final PDWS (EPA, 1977) \\
\hline$\mu g / L$ & 50 & 100 & EPA Method 8270 \\
\hline$\mu \mathrm{g} / \mathrm{L}$ & 50 & 100 & EPA Method 8270 \\
\hline$\mu g / L$ & 50 & 100 & EPA Method 8270 \\
\hline$\mu g / L$ & 2,000 & 4,000 & Final PDWS (EPA, 1993a) \\
\hline$\mu g / L$ & 350 & 700 & Final PDWS (EPA, 1993a) \\
\hline $\mathrm{pCi} / \mathrm{L}$ & $7.5 \mathrm{E}+00$ & $1.5 \mathrm{E}+01$ & Final PDWS (EPA, 1993a) \\
\hline$\mu \mathrm{g} / \mathrm{L}$ & 0.2 & 0.4 & Finar PDWS (EPA, 1993a) \\
\hline$\mu g / L$ & 0.1 & 0.2 & Final PDWS (EPA, 1993a) \\
\hline$\mu g / L$ & 0.00325 & 0.0065 & EPA Method 8280 \\
\hline$\mu \mathrm{g} / \mathrm{L}$ & 0.00325 & 0.0065 & EPA Method 8280 \\
\hline$\mu \mathrm{g} / \mathrm{L}$ & 0.00225 & 0.0045 & EPA Method 8280 \\
\hline$\mu \mathrm{g} / \mathrm{L}$ & 0.00225 & 0.0045 & EPA Method 8280 \\
\hline$\mu \mathrm{g} / \mathrm{L}$ & 0.5 & 1 & Final PDWS (EPA, 1993a) \\
\hline$\mu \mathrm{g} / \mathrm{L}$ & 50 & 100 & EPA Method 8270 \\
\hline$\mu \mathrm{g} / \mathrm{L}$ & 25 & 50 & Final PDWS (EPA, 1993a) \\
\hline$\mu \mathrm{g} / \mathrm{L}$ & 0.00225 & 0.0045 & EPA Method 8280 \\
\hline$\mu g / L$ & 0.00225 & 0.0045 & EPA Method 8280 \\
\hline$\mu \mathrm{g} / \mathrm{L}$ & 0.002 & 0,004 & EPA Method 8280 \\
\hline$\mu g / L$ & 0.002 & 0.004 & EPA Method 8280 \\
\hline$\mu g / L$ & 50 & 100 & EPA Method 8270 \\
\hline$\mu g / L$ & 250 & 500 & EPA Method 8270 \\
\hline$\mu g / L$ & 50 & 100 & EPA Method 8270 \\
\hline$\mu g / L$ & 50 & 100 & EPA Method 8240 \\
\hline$\mu \mathrm{g} / \mathrm{L}$ & 50 & 100 & EPA Method 8270 \\
\hline$\mu \mathrm{g} / \mathrm{L}$ & 250 & 500 & APHA Method 415A \\
\hline $\mathrm{pCi} / \mathrm{L}$ & $5 E-01$ & $1 E+00$ & Interim Final PDWS (EPA, 1977) \\
\hline pCi/L & $1.5 \mathrm{E}+00$ & $3 E+00$ & Interim Final PDWS (EPA, 1977) \\
\hline$\mu \mathrm{g} / \mathrm{L}$ & 75 & 150 & EPA Method 8240 \\
\hline$\mu \mathrm{g} / \mathrm{L}$ & 150 & 300 & SDWS (EPA, 1993b) \\
\hline$\mu \mathrm{g} / \mathrm{L}$ & 150 & 300 & SDWS (EPA, 1993b) \\
\hline$\mu g / L$ & $\begin{array}{l}150 \\
1 F+03\end{array}$ & 300 & $\begin{array}{l}\text { SDWS (EPA, 1993b) } \\
\text { (nterim Final }\end{array}$ \\
\hline pCi/L & $1 E+03$ & $2 \mathrm{E}+03$ & Interim Final PDWS (EPA, 1977) \\
\hline
\end{tabular}


Iron-59c

Isobutyl alcohol

Isodrin.

Isophorone

Isosafrole

Kepone

Lanthanum-140

Lead

Lead, dissolved

Lead, total recoverable

Lead-212

Lindane

Lithium

Lithium, dissolved .

Lithium, total recoverable

Magnesium

Magnesium, dissolved

Magnesium, total recoverable

Manganese

Manganese, dissolved

Manganese, total recoverable

Manganese-54

Mercury

Mercury, dissolved

Mercury, total recoverable

Methacrylonitrile

Methapyrilene

Methoxychlor

3-Methylcholanthrene

2-Methyl-4,6-dinitrophenol

Methyl ethyl ketone

Methyl isobutyl ketone

Methyl methacrylate

Methyl methanesulfonate

2-Methylnaphthalene

Molybdenum

Molybdenum, dissolved

Molybdenum, total recoverable

Naphthalene

1,4-Naphthoquinone

1-Naphthylamine

2-Naphthylamine

Neptunium-237

Nickel

Nickel, dissolved

Nickel, total recoverable

Nickel-59

Nickel-63

Niobium-95'

Nitrate as nitrogen

Nitrate-nitrite as nitrogen

Nitrite as nitrogen

m-Nitroaniline

o-Nitroaniline

p-Nitroaniline

\begin{tabular}{|c|c|c|c|}
\hline pCi/L & $1 E+02$ & $2 E+02$ & Interim Final PDWS (EPA, 1977) \\
\hline$\mu g / L$ & 500 & 1,000 & EPA Method 8240 \\
\hline$\mu g / L$ & 50 & 100 & EPA Method 8270 \\
\hline$\mu g / L$ & 50 & 100 & EPA Method 8270 \\
\hline$\mu \mathrm{g} / \mathrm{L}$ & 50 & 100 & EPA Method 8270 \\
\hline$\mu \mathrm{g} / \mathrm{L}$ & 50 & 100 & EPA Method 8270 \\
\hline $\mathrm{pCi} / \mathrm{L}$ & $3 E+01$ & $6 E+01$ & Interim Final PDWS (EPA, 1977) \\
\hline$\mu g / L$ & 25 & 50 & Final PDWS (SCDHEC, 1981) \\
\hline$\mu g / L$ & 25 & 50 & Final PDWS (SCDHEC, 1981) \\
\hline$\mu g / L$ & 25 & 50 & Final PDWS (SCDHEC, 1981) \\
\hline $\mathrm{pCi} / \mathrm{L}$ & $6.2 E+01$ & $1.23 \mathrm{E}+02$ & Proposed PDWS (EPA, 1991) \\
\hline$\mu g / L$ & 0.1 & 0.2 & Final PDWS (EPA, 1993a) \\
\hline$\mu g / L$ & 25 & 50 & EPA Method 6010 \\
\hline$\mu g / L$ & 25 & 50 & EPA Method 6010 \\
\hline \multirow[t]{4}{*}{$\mu g / L$} & 25 & 50 & EPA Method 6010 \\
\hline & No flag & No flag & Set by EPD/EMS \\
\hline & No flag & No flag & Set by EPD/EMS \\
\hline & No flag & No flag & Set by EPD/EMS \\
\hline$\mu \mathrm{g} / \mathrm{L}$ & 25 & 50 & SDWS (EPA, 1993b) \\
\hline$\mu g / L$ & 25 & 50 & SDWS (EPA, 1993b) \\
\hline$\mu g / L$ & 25 & 50 & SDWS (EPA, 1993b) \\
\hline $\mathrm{pCi} / \mathrm{L}$ & $1.5 E+02$ & $3 E+02$ & Interim Final PDWS (EPA, 1977) \\
\hline$\mu g / L$ & 1 & 2 & Final PDWS (EPA; 1993a) \\
\hline$\mu g / L$ & 1 & 2 & Final PDWS (EPA, 1993a) \\
\hline$\mu g / L$ & 1 & 2 & Final PDWS (EPA, 1993a) \\
\hline$\mu g / L$ & 250 & 500 & EPA Method 8240 \\
\hline$\mu \mathrm{g} / \mathrm{L}$ & $50^{\circ}$ & 100 & EPA Method 8270 \\
\hline$\mu g / L$ & 20 & 40 & Final PDWS (EPA, 1993a) \\
\hline$\mu \mathrm{g} / \mathrm{L}$ & 50 & 100 & EPA Method 8270 \\
\hline \multirow[t]{3}{*}{$\mu g / L$} & 250 & 500 & EPA Method 8270 \\
\hline & No flag & No flag & Set by EPD/EMS \\
\hline & No flag & No flag & Set by EPD/EMS \\
\hline$\mu g / L$ & 50 & 100 & EPA Method 8270 \\
\hline$\mu g / L$ & 50. & 100 & EPA Method 8270 \\
\hline$\mu g / L$ & 50 & 100 & EPA Method 8270 \\
\hline$\mu g / L$ & 250 & 500 . & EPA Method 6010 \\
\hline$\mu g / L$ & 250 & 500 & EPA Method 6010 \\
\hline$\mu g / L$ & 250 & 500 & EPA Method 6010 \\
\hline$\mu g / L$ & 50 & 100 & EPA Method 8270 \\
\hline$\mu g / L$ & 50 & 100 & EPA Method 8270 \\
\hline$\mu \mathrm{g} / \mathrm{L}$ & 50 & 100 & EPA Method 8270 \\
\hline$\mu g / L$ & 50 & 100 & EPA Method 8270 \\
\hline pCi/L & $3.53 E+00$ & $7.06 \mathrm{E}+00$ & Proposed PDWS (EPA, 1991) \\
\hline$\mu g / L$ & 50 & 100 & Final PDWS (EPA, 1993a) \\
\hline$\mu g / L$ & 50 & 100 & Final PDWS (EPA, 1993a) \\
\hline$\mu g / L$ & 50 & 100 & Final PDWS (EPA, 1993a) \\
\hline $\mathrm{pCi} / \mathrm{L}$ & $1.5 E+02$ & $3 E+02$ & Interim Final PDWS (EPA, 1977) \\
\hline pCi/L & $2.5 \mathrm{E}+01$ & $5 E+01$ & Interim Final PDWS (EPA, 1977) \\
\hline $\mathrm{pCi} / \mathrm{L}$ & $1.5 \mathrm{E}+02$ & 3.E+02 & Interim Final PDWS (EPA, 1977) \\
\hline$\mu g / L$ & 5,000 & 10,000 & Final PDWS (EPA, 1993a) \\
\hline$\mu \mathrm{g} / \mathrm{L}$ & 5,000 & 10,000 & Final PDWS (EPA, 1993a) \\
\hline$\mu g / L$ & 500 & 1,000 & Final PDWS (EPA, 1993a) \\
\hline$\mu g / L$ & 50 & 100 & EPA Method 8270 \\
\hline$\mu g / L$ & 50 & 100 & EPA Method 8270 \\
\hline$\mu g / L$ & 50 & 100 & EPA Method 8270 \\
\hline
\end{tabular}


Nitrobenzene

Nitrogen by Kjeldahl method

2-Nitrophenol

4-Nitrophenol

4-Nitroquinoline-1-oxide

N-Nitrosodi-n-butylamine

N-Nitrosodiethylamine

$\mathrm{N}-\mathrm{Nitrosodimethylamine}$

$\mathrm{N}$-Nitrosodiphenylamine

N-Nitrosodipropylamine

N-Nitrosomethylethylamine :

$\mathrm{N}$-Nitrosomorpholine

N-Nitrosopiperidine

$\mathrm{N}$-Nitrosopyrrolidine

5-Nitro-o-toluidine

Nonvolatile beta

Octachlorodibenzo-p-dioxin

isomers

Octachlorodibenzo-p-furan

isomers

Odor

Oil \& Grease

Oxamylb

Parathion

Parathion methyl

PCB 1016

PCB 1221 .

PCB 1232

PCB 1242

PCB 1248

PCB 1254

PCB 1260

PCB 1262

Pentachlorobenzene

Pentachlorodibenzo-p-dioxin

isomers

1,2,3,7,8-PCDD

Pentachlorodibenzo-p-furan

isomers

1,2,3,7,8-PCDF

Pentachloroethane

Pentachloronitrobenzene

Pentachlorophenol

$\mathrm{pH}$

$\mathrm{pH}$

Phenacetin

Phenanthrene

Phenol

Phenols

p-Phenylenediamine

Phorate

Picloramb

2-Picoline

Plutonium-238

Plutonium-239

\begin{tabular}{|c|c|c|c|}
\hline$\mu g / L$ & 50 & 100 & EPA Method 8270 \\
\hline$\mu \mathrm{g} / \mathrm{L}$ & 500 & 1,000 & EPA Method 351.2 \\
\hline$\mu \mathrm{g} / \mathrm{L}$ & 50 & 100 & EPA Method 8270 \\
\hline$\mu g / L$ & 50 & 100 & EPA Method 8270 \\
\hline$\mu g / L$ & 50 & 100 & EPA Method 8270 \\
\hline$\mu \mathrm{g} / \mathrm{L}$ & 50 & 100 & EPA Method 8270 \\
\hline$\mu g / L$ & 50 & 100 & EPA Method 8270 \\
\hline$\mu g / L$ & 50 & 100 & EPA Method 8270 \\
\hline$\mu \mathrm{g} / \mathrm{L}$ & 50 & 100 & EPA Method 8270 \\
\hline$\mu \mathrm{g} / \mathrm{L}$ & 50 & 100 & EPA Method 8270 \\
\hline$\mu g / L$ & 50 & 100 & EPA Mețhod 8270 \\
\hline$\mu g / L$ & 50 & 100 & EPA Method 8270 \\
\hline$\mu g / L$ & 50 & 100 & EPA Method 8270 \\
\hline$\mu g / L$ & 50 & 100 & EPA Method 8270 \\
\hline$\mu \mathrm{g} / \mathrm{L}$ & 50 & 100 & EPA Method 8270 \\
\hline $\mathrm{pCi} / \mathrm{L}$ & $2.5 E+01$ & $5 E+01$ & Interim Final PDWS (EPA, 1977) \\
\hline$\mu \mathrm{g} / \mathrm{L}$ & 0.005 & 0.01 & EPA Method 8280 \\
\hline$\mu \mathrm{g} / \mathrm{L}$ & 0.005 & 0.01 & EPA Method 8280 \\
\hline & No flag & - No flag & Set by EPD/EMS \\
\hline$\mu \mathrm{g} / \mathrm{L}$ & 5,000 & 10,000 & EPA Method 413.1 \\
\hline$\mu \mathrm{g} / \mathrm{L}$ & 100 & 200 & Final PDWS (EPA, 1993a) \\
\hline$\mu g / L$ & 0.25 & 0.5 & EPÁ Method 8080 \\
\hline$\mu g / L$ & 0.25 & 0.5 & EPA Method 8080 \\
\hline$\mu \mathrm{g} / \mathrm{L}$ & 0.25 & 0.5 & Final PDWS (EPA, 1993a) \\
\hline$\mu \mathrm{g} / \mathrm{L}$ & 0.25 & 0.5 & Final PDWS (EPA, 1993a) \\
\hline$\mu g / L$ & 0.25 & 0.5 & Final PDWS (EPA, 1993a) \\
\hline$\mu \mathrm{g} / \mathrm{L}$ & 0.25 & 0.5 & Final PDWS (EPA, 1993a) \\
\hline$\mu \mathrm{g} / \mathrm{L}$ & 0.25 & 0.5 & Final PDWS (EPA, 1993a) \\
\hline$\mu \mathrm{g} / \mathrm{L}$ & 0.25 & 0.5 & Final PDWS (EPA, 1993a) \\
\hline$\mu \mathrm{g} / \mathrm{L}$ & 0.25 & 0.5 & Final PDWS (EPA, 1993a) \\
\hline$\mu \mathrm{g} / \mathrm{L}$ & 0.25 & 0.5 & Final PDWS (EPA, 1993a) \\
\hline$\mu \mathrm{g} / \mathrm{L}$ & 50 & 100 & EPA Method 8270 \\
\hline$\mu g / L$ & 0.00275 & 0.0055 & EPA Method 8280 \\
\hline$\mu \mathrm{g} / \mathrm{L}$ & 0.00275 & 0.0055 & EPA Method 8280 \\
\hline$\mu g / L$ & 0.00275 & 0.0055 & EPA Method 8280 \\
\hline$\mu \mathrm{g} / \mathrm{L}$ & 0.00275 & 0.0055 & EPA Method 8280 \\
\hline$\mu g / L$ & 50 & 100 & EPA Method 8270 \\
\hline$\mu g / L$ & 50 & 100 & EPA Method 8270 \\
\hline$\mu \mathrm{g} / \mathrm{L}$ & 0.5 & 1 & Final PDWS (EPA, 1993a) \\
\hline $\mathrm{pH}$ & 8 & 10 & Set by EPD/EMS \\
\hline $\mathrm{pH}$ & 4 & 3 & Set by EPD/EMS \\
\hline$\mu g / L$ & 50 & 100 & EPA Method 8270 \\
\hline$\mu g / L$ & 50 & 100 & EPA Method 8270 \\
\hline$\mu g / L$ & 50 & 100 & EPA Method 8270 \\
\hline$\mu g / L$ & 25 & 50 & EPA Method 420.1 \\
\hline$\mu \mathrm{g} / \mathrm{L}$ & 50 & 100 & EPA Method 8270 \\
\hline$\mu g / L$ & 0.5 & 1 & EPA Method 8080 \\
\hline$\mu g / L$ & 250 & 500 & Final PDWS (EPA, 1993a) \\
\hline$\mu \mathrm{g} / \mathrm{L}$ & 50 & 100 & EPA Method 8270 \\
\hline $\mathrm{pCi} / \mathrm{L}$ & $3.51 E+00$ & $7.02 E+00$ & Proposed PDWS (EPA, 1991) \\
\hline $\mathrm{pCi} / \mathrm{L}$ & $3.11 E+01$ & $6.21 E+01$ & Proposed PDWS (EPA, 1991) \\
\hline
\end{tabular}


Plutonium-239/240

Plutonium-240

Plutonium-241c

Plutonium-242

Potassium

Potassium, dissolved

Potassium, total recoverable

Potassium-40

Promethium-144

Promethium-146

Promethium-147

Pronamid

Propionitrile

Pyrene

Pyridine

Radium (alpha-emitting) ${ }^{f}$

Radium-226

Radium-228

Radon-222

Ruthenium-103c

Ruthenium-106

Safrole

Selenium

Selenium, dissolved

Selenium, total recoverable

Silica

Silica, dissolved

Silica, total recoverable

Silver

Silver, dissolved

Silver, total recoverable

Simazine ${ }^{b}$

Sodium

Sodium, dissolved

Sodium, total recoverable

Sodium-22

Specific c̀onductance

Strontium-89

Strontium-89/90e

Strontium-90

Styrene

Sulfate

Sulfide

Sulfotepp

Surfactants

2,3,7,8-TCDD

2,3,7,8-TCDF

Technetium-99

1,2,4,5-Tetrachlorobenzene

Tetrachlorodibenzo-p-dioxin

isomers

Tetrachlorodibenzo-p-furan isomers

1,1,1,2-Tetrachloroethane

1,1,2,2-Tetrachloroethane

Tetrachloroethylene

\begin{tabular}{|c|c|c|c|}
\hline $\mathrm{pCi} / \mathrm{L}$ & $3.11 E+01$ & $6.21 E+01$ & Proposed PDWS (EPA, 1991) \\
\hline $\mathrm{pCi} / \mathrm{L}$ & $3.11 \mathrm{E}+01$ & $6.22 E+01$ & Proposed PDWS (EPA, 1991) \\
\hline $\mathrm{Ci} / \mathrm{L}$ & $3.13 E+01$ & $6.26 E+01$ & Proposed PDWS (EPA, 1991) \\
\hline $\mathrm{Ci} / \mathrm{L}$ & 3.27E+01 & $6.54 E+01$ & Proposed PDWS (EPA, 1991) \\
\hline & No flag & No flag & Set by EPD/EMS \\
\hline & No flag & No flag & Set by EPD/EMS \\
\hline & No flag. & No flag & Set by EPD/EMS \\
\hline $\mathrm{Ci} / \mathrm{L}$ & $1.5 E+02$ & $3 E+02$ & Proposed PDWS (EPA, 1986) \\
\hline Ci/L & $5 E+01$ & $1 E+02$ & EPA Method 901.1 \\
\hline $\mathrm{Ci} / \mathrm{L}$ & $5 E+01$ & $1 E+02$ & EPA Method 901.1 \\
\hline $\mathrm{Ci} / \mathrm{L}$ & $2.62 E+03$ & $5.24 E+03$ & Proposed PDWS (EPA, 1991) \\
\hline$g / L_{1}$ & 50 & 100 & EPA Method 8270 \\
\hline$\mu g / L$ & 1,000 & 2,000 & EPA Method 8240 \\
\hline$\mu g / L$ & 50 & 100 & EPA Method 8270 \\
\hline$/ \mathrm{L}$ & 50 & 100 & EPA Method 8270 \\
\hline pCi/L & $1 E+01$ & $2 E+01$ & Proposed PDWS (EPA, 1991) \\
\hline Ci/L & $1 E+01$ & $2 E+01$ & Proposed PDWS (EPA, 1991) \\
\hline Cill & 1E+01 & $2 E+01^{\circ}$ & Proposed PDWS (EPA, 1991) \\
\hline $\mathrm{Ci} / \mathrm{C}^{\circ}$ & $1.5 E+02$ & $3 E+02$ & Proposed PDWS (EPA, 1991) \\
\hline $\mathrm{Ci} / \mathrm{L}$ & $1 E+02$ & $2 E+02$ & Interim Final PDWS (EPA, 1977) \\
\hline $\mathrm{Oi} / \mathrm{L}$ & $1.5 E \div 01$ & $3 E+01$ & Interim Final PDWS (EPA, 1977) \\
\hline$/ / L$ & 50 & 100 & EPA Method 8270 \\
\hline & 25 & 50 & Final PDWS (EPA, 1993a) \\
\hline$d /$ & 25 & 50 & Final PDWS (EPA, 1993a) \\
\hline$y / L$ & 25 & 50 & Final PDWS (EPA, 1993a) \\
\hline & No flag & No flag & Set by EPD/EMS \\
\hline & No flag & No flag & Set by EPD/EMS \\
\hline & No flag & No flag & Set by EPD/EMS \\
\hline$/ L$ & 50 & 100 & SDWS (EPA, 1993b) \\
\hline /L & 50 & 100 & SDWS (EPA, 1993b) \\
\hline & 50 & 100 & SDWS (EPA, 1993b) \\
\hline$y / L$ & 2 & 4 & Final PDWS (EPA, 1993a) \\
\hline & No flag & No flag & Set by EPD/EMS \\
\hline & No flag & No flag & Set by EPD/EMS \\
\hline & No flag & No flag & Set by EPD/EMS \\
\hline Bill & 2.33E +02 & $4.66 \mathrm{E}+02$ & Proposed PDWS (EPA, 1991) \\
\hline $3 / \mathrm{cm}$ & $250^{\prime}$ & 500 & Set by EPD/EMS \\
\hline $\mathrm{Ci} / \mathrm{L}$ & $1 E+01$ & $2 E+01$ & Interim Final PDWS (EPA, 1977) \\
\hline $\mathrm{Bi} / \mathrm{L}$ & $4 E+00$ & $8 E+00$ & Final PDWS (EPA, 1993a) \\
\hline $\mathrm{Bi} / \mathrm{L}$ & $4 E+00$ & $8 E+00$ & Final PDWS (EPA, 1993a) \\
\hline & 50 & 100 & Final PDWS (EPA, 1993a) \\
\hline /L & 200,000 & 400,000 & Proposed PDWS (EPA, 1990) \\
\hline & 5,000 & 10,000 & EPA Method 9030 \\
\hline $7 / 1$ & 50 & 100 & EPA Method 8270 \\
\hline & No flag & No flag & Set by EPD/EMS \\
\hline & 0.000015 & 0.00003 & Final PDWS (EPA, 1993a) \\
\hline$y$ & 0.002 & 0.004 & EPA Method 8280 \\
\hline $\mathrm{pCi} / \mathrm{L}$ & $4.5 E+02$ & $9 E+02$ & Interim Final PDWS (EPA, 1977) \\
\hline & 50 & 100 & EPA Method 8270 \\
\hline$\mu \mathrm{g} / \mathrm{L}$ & 0.00225 & 0.0045 & EPA Method 8280 \\
\hline 作 & 0.002 & 0.004 & EPA Method 8280 \\
\hline & 5 & 10 & EPA Method 8240 \\
\hline$\mu g / L$ & 5 & 10 & EPA Method 8240 \\
\hline$\mu \mathrm{g} / \mathrm{L}$ & 2.5 & 5 & Final PDWS (EPA, 1993a) \\
\hline
\end{tabular}


2,3,4,6-Tetrachlorophenol

Thallium

Thallium, dissolved

Thallium, total recoverable

Thionazin

Thorium-228

Thorium-230

Thorium-232

Thorium-234

Tin

Tin, dissolved

Tin, total recoverable

Tin- $113^{\circ}$

Toluene

o-Toluidine

Total carbon

Total coliform

Total dissolved solids

Total hydrocarbons

Total inorganic carbon

Total organic carbon

Total organic halogens

Total organic nitrogen

Total petroleum hydrocarbons

Total phosphates (as P)

Total phosphorus

Toxaphene

2,4,5-TP (Silvex)

Tributyl phosphate

1,2,4-Trichlorobenzene

1,1,1-Trichloroethane

1,1,2-Trichloroethane

Trichloroethylene

Trichlorofluoromethane

2,4,5-Trichlorophenol

2,4,6-Trichlorophenol

2,4,5-Trichlorophenoxyacetic acid

1,2,3-Trichloropropane

$0,0,0$-Triethyl phosphorothioate

1,3,5-Trinitrobenzene

Tritium

Turbidity 9

Uranium

Uranium, dissolved

Uranium, total recoverable

Uranium alpha activity

Uranium-233/234

Uranium-234

Uranium-235

Uranium-238

Vanadium

Vanadium, dissolved

Vanadium, total recoveräble

Vinyl acetate

Xylenes

\begin{tabular}{|c|c|c|c|}
\hline$\mu g / L$ & 50 & 100 & EPA Method 8270 \\
\hline$\mu g / L$ & 1 & 2 & Final PDWS (EPA, 1993a) \\
\hline$\mu g / L$ & 1 & 2 & Final PDWS (EPA, 1993a) \\
\hline$\mu g / L$ & 1 & 2 & Final PDWS (EPA, 1993a) \\
\hline$\mu g / L$ & 50 & 100 & EPA Method 8270 \\
\hline pCi/L & $6.25 E+01$ & $1.25 E+02$ & Proposed PDWS (EPA, 1991) \\
\hline $\mathrm{Ci} / \mathrm{L}$ & $3.96 \mathrm{E}+01$ & $7.92 E+01$ & Proposed PDWS (EPA, 1991) \\
\hline $\mathrm{Ci} / \mathrm{L}$ & $4.4 E+01$ & $8.8 E+01$ & Proposed PDWS (EPA, 1991) \\
\hline $\mathrm{Ci} / \mathrm{L}$ & $2 E+02$ & 4.01E+02 & Proposed PDWS (EPA, 1991) \\
\hline$g / L$ & 10 & 20 & EPA Method 282.2 \\
\hline$g / L$ & 10 & 20 & EPA Method 282.2 \\
\hline$g / L$ & 10 & 20 & EPA Method 282.2 \\
\hline $\mathrm{Ci} / \mathrm{L}$ & $1.5 E+02$ & $3 E+02$ & Interim Final PDWS (EPA, 1977) \\
\hline$g / L$ & 500 & 1,000 & Final PDWS (EPA, 1993a) \\
\hline$g / L$ & 50 & 100 & EPA Method 8270 \\
\hline$g / L$ & 5,000 & 10,000 & EPA Method 9060 \\
\hline & 0 & 0 & Final PDWS (EPA, 1993a) \\
\hline & No flag & No flag & Set by EPD/EMS \\
\hline$g / L$ & 5,000 & 10,000 & EPA Method 418.1 \\
\hline$g / L$ & 5,000 & 10,000 & EPA Method 9060 \\
\hline$g / L$ & 5,000 & 10,000 & EPA Method 9060 \\
\hline$\mu g / L$ & 25 & 50 & EPA Method 9020 \\
\hline$g / L$ & 500 & 1,000 & APHA Method 420 \\
\hline$g / L$ & 5,000 & 10,000 & EPA Method 418.1 \\
\hline & No flag & No flag & Set by EPD/EMS \\
\hline & No flag & No flag & Set by EPD/EMS \\
\hline$\mu g / L$ & 1.5 & 3 & Final PDWS (EPA, 1993a) \\
\hline$g / L$ & 25 & 50 & Final PDWS (EPA, 1993a) \\
\hline$g / L$ & 50 & 100 & EPA Method 8270 \\
\hline$g / L$ & 35 & 70 & Final PDWS (EPA, 1993a) \\
\hline$\mu g / L$ & 100 & 200 & Final PDWS (EPA, 1993a) \\
\hline$g / L$ & 2.5 & 5 & Final PDWS (EPA, 1993a) \\
\hline$g / L$ & 2.5 & 5. & Final PDWS (EPA, 1993a) \\
\hline$\mu g / L$ & 5 & 10 & EPA Method 8240 \\
\hline$g / L$ & 50 & 100 & EPA Method 8270 \\
\hline$\mu g / L$ & 50 & 100. & EPA Method 8270 \\
\hline$\mu g / L$ & 2.5 & 5 & EPA Method 8150 \\
\hline$\mu g / L$ & 5 & 10 & EPA Method 8240 \\
\hline$\mu g / L$ & 50 & 100 & EPA Method 8270 \\
\hline$\mu g / L$ & 50 & 100 & EPA Method 8270 \\
\hline $\mathrm{pCi} / \mathrm{mL}$ & $\begin{array}{l}1 E+01 \\
\text { No flag }\end{array}$ & $\begin{array}{l}2 E+01 \\
\text { No flag. }\end{array}$ & $\begin{array}{l}\text { Final PDWS (EPA, 1993a) } \\
\text { Set by EPD/EMS }\end{array}$ \\
\hline$\mu g / L$ & 10 & 20 & Proposed PDWS (EPA, 1991) \\
\hline$\mu g / L$ & 10 & 20 & Proposed PDWS (EPA, 1991) \\
\hline$\mu g / L$ & 10 & 20 & Proposed PDWS (EPA, 1991) \\
\hline $\mathrm{pCi} / \mathrm{L}^{\prime}$ & $1.5 E+01$ & $3 E+01$ & Proposed PDWS (EPA, 1991) \\
\hline $\mathrm{pCi} / \mathrm{L}$ & $6.9 E+00$ & $1.38 \mathrm{E}+01$ & Proposed PDWS (EPA, 1991) \\
\hline $\mathrm{pCi} / \mathrm{L}$ & $6.95 E+00$ & $1.39 E+01$ & Proposed PDWS (EPA, 1991) \\
\hline $\mathrm{pCi} / \mathrm{L}$ & $7.25 E+00$ & $1.45 E+01$ & Proposed PDWS (EPA, 1991) \\
\hline $\mathrm{pCi} / \mathrm{L}$ & $7.3 E+00$ & $1.46 \mathrm{E}+01$ & Proposed PDWS (EPA, 1991) \\
\hline$\mu g / L$ & 40 & 80 & EPA Method 6010 \\
\hline$\mu g / L$ & 40 & 80 & EPA Method 6010 \\
\hline$\mu \mathrm{g} / \mathrm{L}$ & 40 & 80 & EPA Method 6010 \\
\hline$\mu g / L$ & 5 & 10 & EPA Method 8240 \\
\hline$\mu g / L$ & 5,000 & 10,000 & Final PDWS (EPA, 1993a) \\
\hline
\end{tabular}


Yttrium-88

Zinc

Zinc, dissolved

Zinc, total recoverable

Zinc-65

Zirconium-95c

Zirconium/Niobium-95c

$\begin{array}{lll}\text { pCi/L } & 5 E+01 & 1 E+02 \\ \mu g / L & 2,500 & 5,000 \\ \mu g / L & 2,500 & 5,000 \\ \mu g / L & 2,500 & 5,000 \\ \text { pCi/L } & 1.5 E+02 & 3 E+02 \\ \text { pCi/L } & 1 E+02 & 2 E+02 \\ \text { pCi/L } & 1 E+02 & 2 E+02\end{array}$

EPA Method 901.1

SDWS (EPA, 1993b)

SDWS (EPA, 1993b)

SDWS (EPA, 1993b)

Interim Final PDWS (EPA, 1977)

Interim Final PDWS (EPA, 1977)

Interim Final PDWS (EPA, 1977)

a References for methods are in Appendix E; references for dated sources are at the end of this appendix.

b EMS is currently unable to perform this analysis.

c EMS discontinued monitoring this radionuclide because it is inappropriate for the SRS Groundwater Monitoring Program.

d EPD/EMS set this flagging criterion using the 1991 proposed PDWS because the final PDWS in 1977 may have been in error.

- For double radionuclide analyses where each separate radionuclide has its own standard, the more stringent standard is used.

$f$ The applied standard is for radium-226.

9 The primary maximum contaminant level range for turbidity is $1-5 \mathrm{NTU}$, which is inappropriate for the SRS Groundwater Monitoring Program.

\section{References}

EPA (U.S. Environmental Protection Agency), 1977. National Interim Primary Drinking Water Regulations, EPA-570/9-76-003. Washington, DC.

EPA (U.S. Ėnvironmental Protection Agency), 1986. Water Pollution Control; National Primary Drinking Water Regulations, Radionuclides (Proposed): Federal Register, September 30, 1986, pp. 34835-34862. Washington, DC.

EPA (U.S. Environmental Protection Agency), 1990. National Primary and Secondary Drinking Water Regulations; Synthetic Organic Chemicals and Inorganic Chemicals (Proposed Rule).

Federal Register, July 25, 1990, pp. 30369-30448. Washington, DC.

EPA (U.S. Environmental Protection Agency), 1991. National Primary Drinking Water Regulations; Radionuclides; Proposed Rule. Federal Register, July 18, 1991, pp. 33052-33127. Washington, DC.

EPA (U.S. Environmental Protection Agency), 1993a. National Primary Drinking Water Regulations. Code of Federal Regulatiońs, Title 40, Part 141, pp. 592-732. Washington, DC.

EPA (U.S. Environmental Protection Agency), 1993b. National Secondary Drinking Water Regulations. Code of Federal Regulations, Title 40, Part 143, pp. 774-777. Washington, DC.

SCDHEC (South Carolina Department of Health and Environmental Control), 1981. State Primary . Drinking Water Regulations, R.61-58.5. Columbia, SC. 


\section{Appendix C}

\section{Figures}


WSRC-TR-95-0143-2

Unclassified

THIS PAGE LEFT BLANK INTENTIONALLY. 


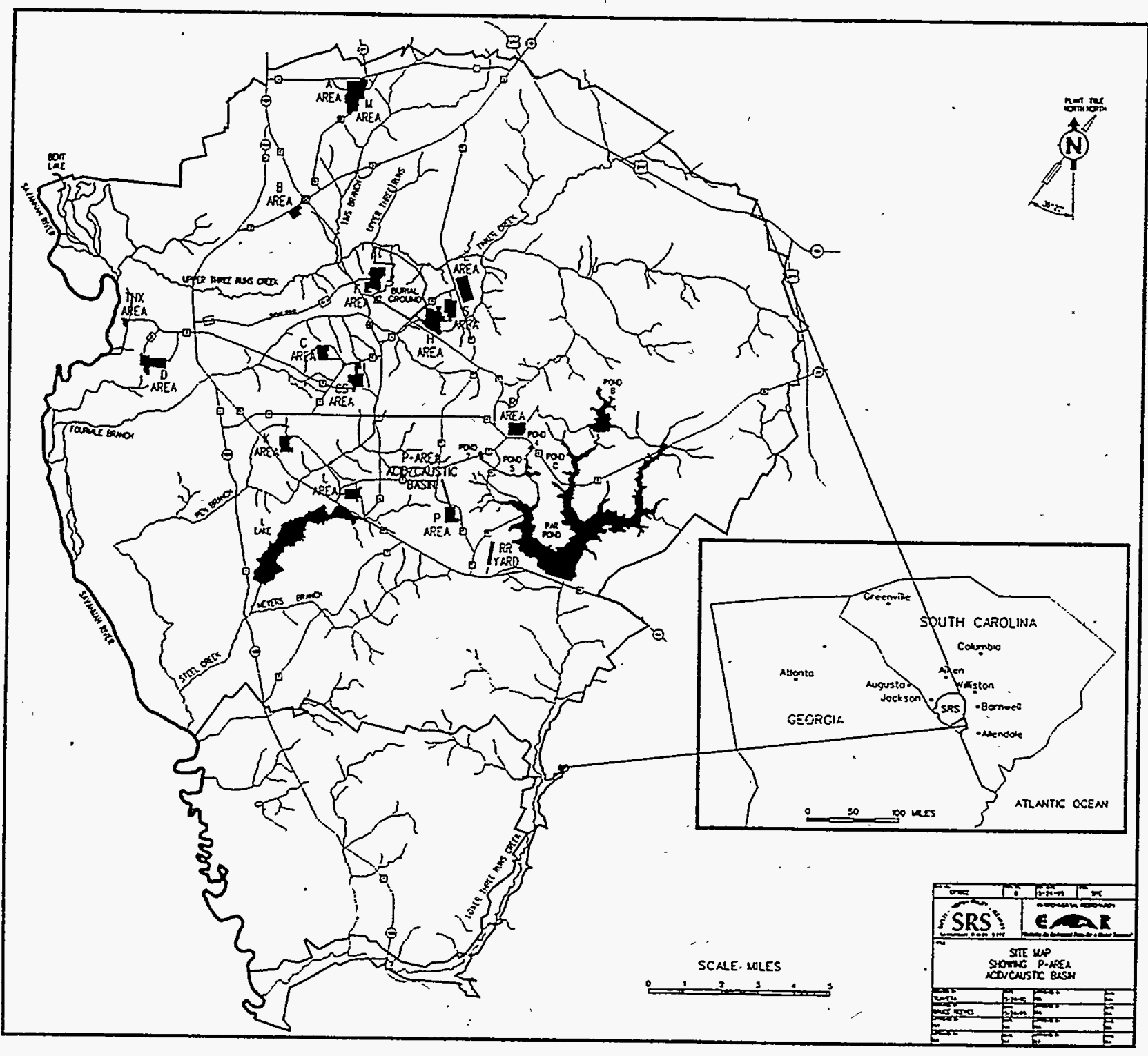

Figure 1. Location of the P-Area Acid/Caustic Basin at the Savannah River Site 


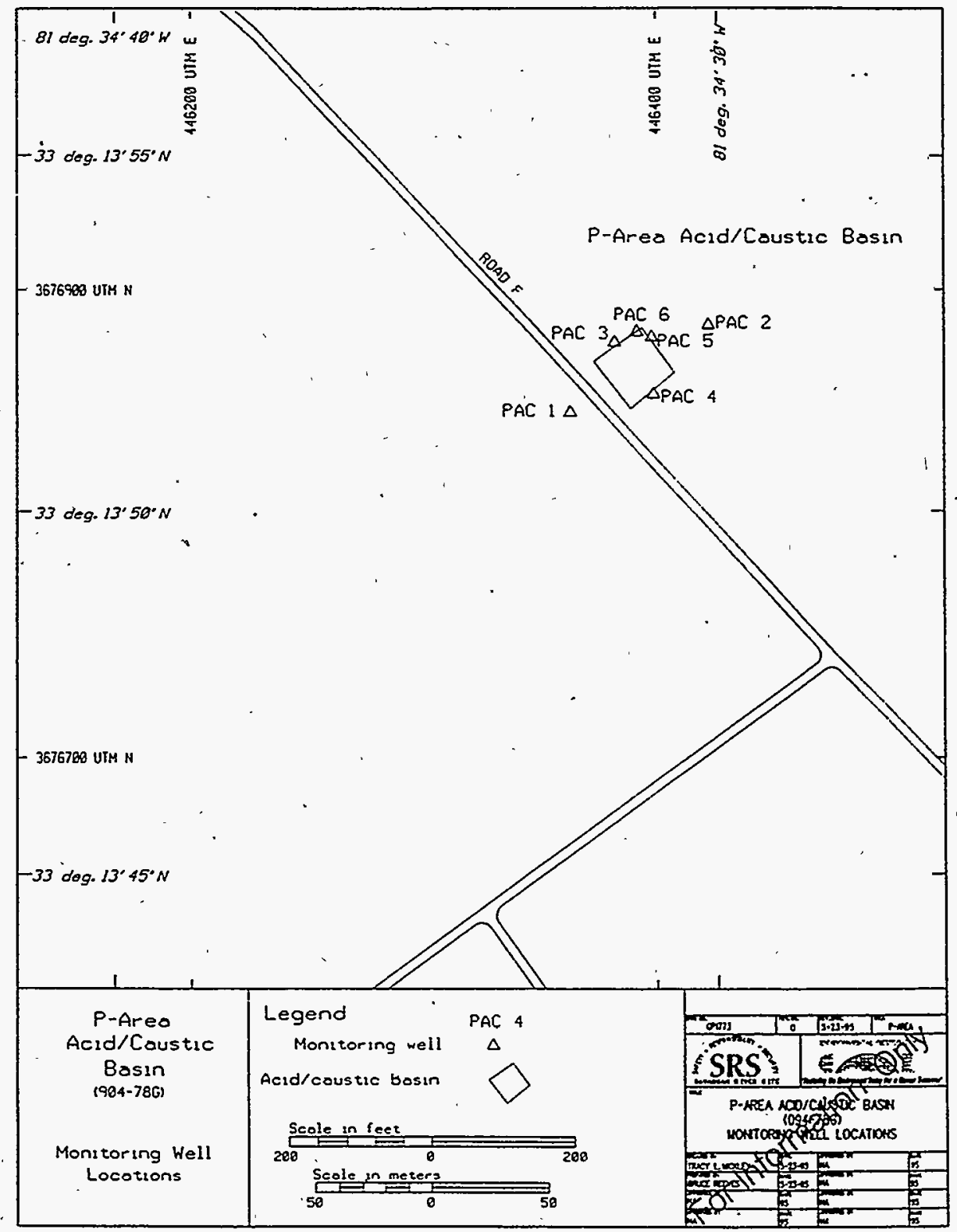

Figure 2. Location of Groundwater Monitoring Wells at the P-Area Acid/Caustic Basin 
u!seg o!!sneว/p!

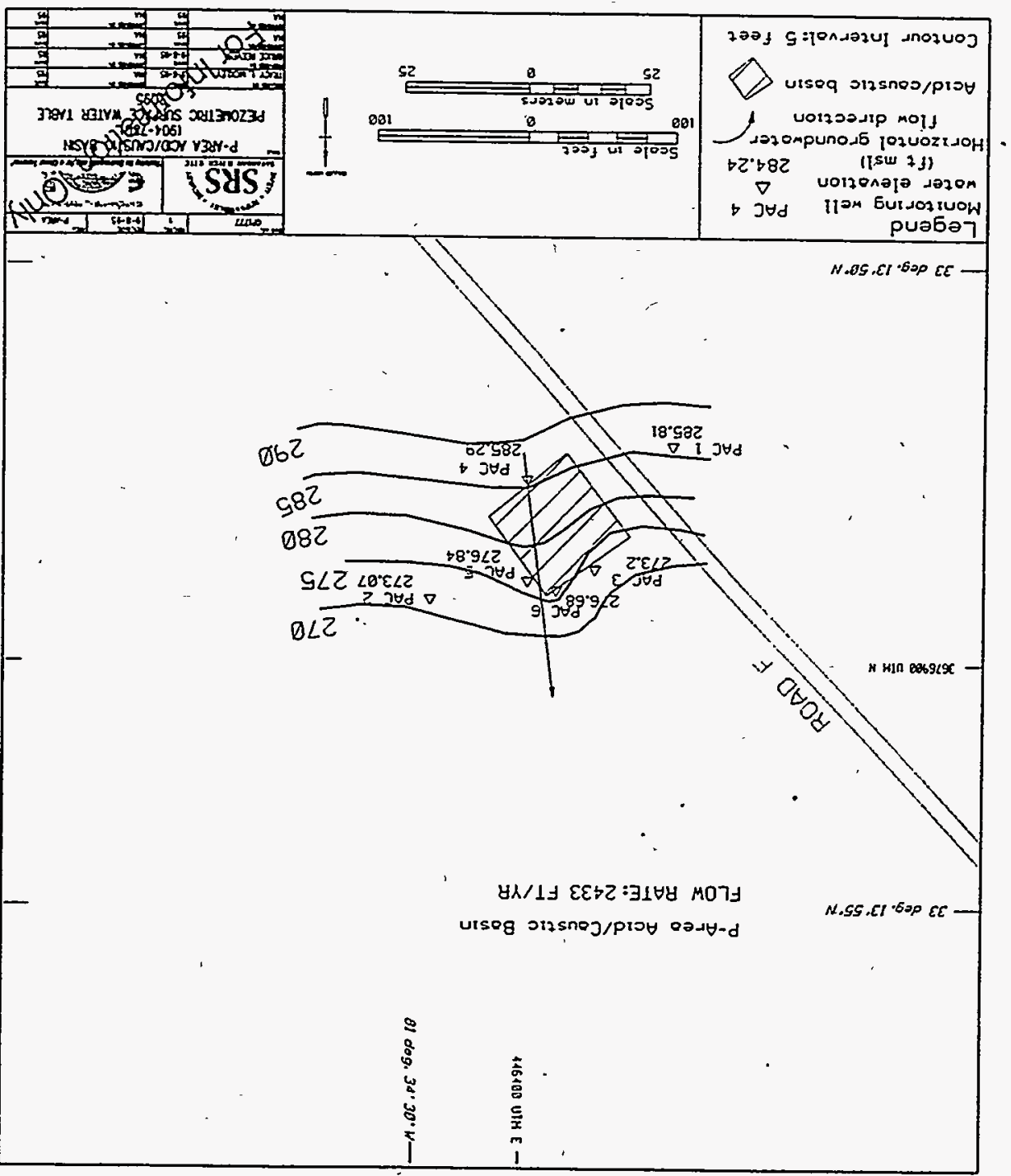


THIS PAGE LEFT BLANK INTENTIONALLY. 


\section{Appendix D}

\section{Groundwater Monitoring Results Tables}


THIS PAGE LEFT BLANK INTENTIONALLY. 


\section{Key to Reading the Tables}

The following abbreviations may appear in the data tables:

\section{Constituents}

$1,2,3,4,6,7,8-H P C D D$ $1,2,3,4,6,7,8$-HPCDF

$1,2,3,4,7,8-H X C D D$ $1,2,3,4,7,8-\mathrm{HXCDF}$ Lindane.

PCB

$1,2,3,7,8-\mathrm{PCDD}$

1,2,3,7,8-PCDF

Sp. conductance

TCDD

TCDF

\section{Laboratories}

CN

EM

GE and GP

SC

SP

TM

WA and WS

\section{Sampling Codes}

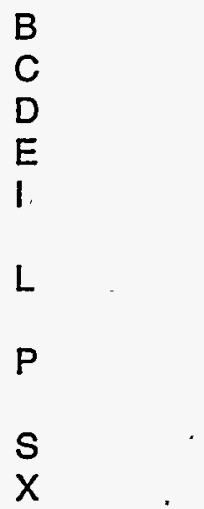

\section{Sampling Methods}

B

$P$

$S$

V $1,2,3,4,6,7,8$-heptachlorodibenzo-p-dioxin

$1,2,3,4,6,7,8$-heptachlorodibenzo-p-furan

$1,2,3,4,7,8$-hexachlorodibenzo-p-dioxin

1,2,3,4,7,8-hexachlorodibenzo-p-furan

gamma-benzene hexachloride

polychlorinated biphenyl

1,2,3,7,8-pentachlorodibenzo-p-dioxin

1,2,3,7,8-pentachlorodibenzo-p-furan

specific conductance

tetrachlorodibenzo-p-dioxin

tetrachlorodibenzo-p-furan

Clemson Technical Center, Inc.

Environmental Protection Department/Environmental . Monitoring Section (EPD/EMS) Laboratory

General Engineering Laboratories

Savannah River Technology Center

Spencer Testing Services, Inc.

TMAVEberline

Roy F. Weston, Inc.

blank sample was collected

well was pumping continuously

well was dry

equipment blank was collected

well went dry during sampling; insufficient water to collect all samples

well went dry before sampling began; only depth to water can be determined

inaccessibility or mechanical failure prevented sample

collection and field analysis of the water

no water in standpipe; for water level events only

well went dry during purging; samples collected after well

recovered

sample collected using an open-bucket bailer

sample collected using a bladder pump

sample collected using a single-speed centrifugal

downhole pump

sample collected using a variable-speed pump

Units 


\section{E}

$\mathrm{mg} / \mathrm{L}$

$\mathrm{msl}$

MSL'

NTU

$\mathrm{pCi} / \mathrm{L}$

$\mathrm{pCi} / \mathrm{mL}$

$\mathrm{pH}$

$\mu \mathrm{g} / \mathrm{L}$

$\mu \mathrm{S} / \mathrm{cm}$ exponential notation (e.g., 1.1E-09 $=1.1 \times 10^{-9}=$ $0.0000000011)^{\circ}$

milligrams per liter mean sea level million structures per liter turbidity unit picocuries per liter picocuries per milliliter $\mathrm{pH}$ unit micrograms per liter. microsiemens per centimeter

Other

CS
DF
H
Mod
PDWS
PVC
ST
TOC

carbon steel

dilution factor column in data tables

holding time column in data tables

modifier column in data tables primary drinking water standard polyvinyl chloride exceeded standard column in data tables top of casing

\section{Holding Times}

Standard analytical methods include a limit, called holding time, on the maximum elapsed time between sample collection and extraction or analysis by the laboratory. In the data tables, a large bullet ( $\bullet$ ) in the $H$ (holding time) column indicates that holding time was exceeded. Analyses performed beyond holding times may not yield valid results.

The South Carolina Department of Health and Environmental Control allows only 15 minutes to elapse between sampling and analysis for $\mathrm{pH}$. Thus, only field $\mathrm{pH}$ measurements can meet the holding time criterion; laboratory $\mathrm{pH}$ anälyses always will exceed it.

The Jaboratory procedure used for the determination of specific conductance allows one day to elapse between sampling and analysis. Thus, laboratory specific conductance measurements may exceed the holding time criterion.

\section{Data Rounding}

Constituent results in analytical results tables that appear to equal the final PDWS but are not marked in the ST (exceeded the final PDWS or screening level) column are below the final PDWS in the database. Values stored in the database contain more significant digits than the reported results. Apparent discrepancies in the tables are due to the rounding of reported results. 


\section{Data Qualification}

The contract laboratories continually assess their own accuracy and precision according to U.S. Environmental Protection Agency (EPA) guidelines. They submit sample- or batch-specific quality assurance/quality control information either at the same time as analytical results or in a quarterly summary. Properly defined and used result modifiers (also referred to as qualifiers) can be a key component in assessing data usability. Result modifiers designed by the EPD/EMS and provided to the primary laboratories are defined below. These modifiers appear in the data tables under the column Mod. The lettered modifiers are based on EPA's STORET codes.

\section{Result modifier}

(Blank)

$J$

1

L

M

$\mathbf{R}$

T

U

V

Y

1

2

3

4

6
Data are not qualified. Numbers should be interpreted exactly as reported.

Value is estimated because quantitation in the sample or in associated quality control samples did not meet specifications.

The value in the result field is the instrument reading, not the sample quantification limit. Always used with the result qualifier $U$.

Value is off-scale high. The actual value is not known but is known to be greater than the value shown.

Presence of the analyte is verified but not quantified.

Result was rejected because performance requirements in the sample analysis or associated quality control analyses were not met.

Analyte was not detected; if present, it was below the criteria for detection.

Material analyzed for but not detected. Analytical result reported is less than the sample quantitation limit.

Analyte was detected in an associated method blank.

Result was obtained from an unpreserved or improperly preserved sample. Data may not be accurate.

Result may be an underestimation of the true value due to analytical bias.

Result may be an overestimation of the true value due to analytical bias.

The associated result may be of poor precision (high variability) due to analytical bias.

Result is associated with QA results indicating matrix interference.

The associated result is from a reanalysis performed out of holding time due to problems with an earlier analysis. 
Table 1. Maximum Results for Constituents Exceeding Final Primary Drinking Water Standards

Well

Constituent

Unit

$\underline{3094}$

$\underline{4094}$

1095

$\underline{2095}$

Mod

$\mathrm{N}^{\mathrm{a}}$

None

N

$\mathrm{N}$

$\mathbf{N}$

$N$

Note: The modifier column applies to second quarter.1995 data only.

a $\mathrm{N}=$ not applicable.

Table 2. Maximum Results for Constituents Exceeding Other Flag 2 Criteria or the SRS Turbidity Standard

\begin{tabular}{|c|c|c|c|}
\hline Well & Constituent & $\underline{\text { Unit }}$ & $\underline{2095}$ \\
\hline PAC 1 & Aluminum, total recoverable & $\mu g / L$ & 124 \\
\hline & & & 144 \\
\hline \multirow{3}{*}{ PAC 2} & & & \\
\hline & Iron, total recoverable & $\mu \mathrm{g} / \mathrm{L}$ & 5440 \\
\hline & Radium-228 & $\mathrm{pCi} / \mathrm{L}$ & 63.7 \\
\hline PAC 3 & Iron, total recoverable & $\mu g / L$ & 1480 \\
\hline \multirow[t]{2}{*}{ PAC 4} & Aluminum, total recoverable & $\mu g / L$ & $\begin{array}{l}126 \\
158\end{array}$ \\
\hline & , & & \\
\hline \multirow[t]{3}{*}{ PAC 5} & Aluminum, total recoverable & $\mu g / L$ & 278 \\
\hline & Iron, total recoverable & $\mu \mathrm{g} / \mathrm{L}$ & 761 \\
\hline & Manganese, total recoverable & $\mu g / L$ & 306 \\
\hline \multirow[t]{3}{*}{ PAC 6} & Aluminum, total recoverable & $\mu \mathrm{g} / \mathrm{L}$ & 186 \\
\hline & Iron, total recoverable. & & 2560 \\
\hline & Manganese, total recoverable & $\mu \mathrm{g} / \mathrm{L}$. & 203 \\
\hline
\end{tabular}

Notes: These results do not include field data. The groundwater samples are unfiltered. Thus, the results for metals are for total recoverable metals. Flags are established by EPD/EMS and are based on final PDWS, Secondary Drinking Water Standards, or method detection limits (Appendix B). 


\section{Table 3. Groundwater Monitoring Results for Individual Wells}

\section{WELL: PAC1}

SRS Coord. Lat/Longitude

Screen Zone Elevation

Top of Casing

Casing Pump

Formation

$\begin{array}{ll}\text { N } 43543.3 & 33.230963 \mathrm{Deg} \mathrm{N} \\ \mathrm{E} 66753.4 & -81.575658 \mathrm{Deg} W\end{array}$ 283.9-253.9 ft msl $295.9 \mathrm{ft} \mathrm{msl}$

4" PVC S

Water Table

\section{FIELD MEASUREMENTS}

Sample date: 04/20/95

Depth to water: $10.09 \mathrm{ft}(3.076 \mathrm{~m})$ below TOC

Water elevation: $285.81 \mathrm{ft}(87.14 \mathrm{~m}) \mathrm{msl}$

Sp. conductance: $37 \mathrm{uS} / \mathrm{cm}$

Turbidity: 5.6 NTU

Water evacuated before sampling: 392 gal

\section{LABORATORY ANALYSES}

H ST Analyte

Gross alpha

Gross alpha

Gross alpha,

Gross alpha

Nitrate as nitrogen

Nitrate as nitrogen

Nitrate-nitrite as nitrogen

Nitrate-nitrite as nitrogen

Nitrate-nitrite as nitrogen

Nonvolatile beta

Nonvolatile beta

Nonvolatile beta

Nonvolatile beta

Tritium

Tritium

Tritium

Tritium

Aluminum, total recoverable

Aluminum, total recoverable

Aluminum, total recoverable

Aluminum, total recoverable

Aluminum, total recoverable

Arsenic, total recoverable

Arsenic, total recoverable

Arsenic, total recoverable

Arsenic, total recoverable

Barium, total recoverable

Barium, total recoverable

Barium, total recoverable

Barium, total recoverable

Barium, total recoverable
Time: $13: 25$

pH:5.4

Alkalinity:4 mg/L

Water temperature: $17.1 \mathrm{deg} C$

Volumes purged: 18.73 well volumes

$\underline{\text { Result }} \underline{D F} \underline{\text { Mod }} \underline{\text { Unit }} \underline{\text { Flag }} \underline{\text { Lab }}$

$\begin{array}{llllll}1.29 & 1 & \mathrm{~J} & \mathrm{pCi} / \mathrm{L} & 0 & \mathrm{GP} \\ <-.0334 & \mathrm{I} & \mathrm{UJI} & \mathrm{pCi} / \mathrm{L} & 0 & \mathrm{GP} \\ <-.5 & 1 & \mathrm{UI} & \mathrm{pCi} / \mathrm{L} & 0 & \mathrm{TM} \\ <-.5 & 1 & \mathrm{UI} & \mathrm{pCi} / \mathrm{L} & 0 & \mathrm{TM} \\ 620 & 1 & & \mathrm{ug} / \mathrm{L} & 0 & \mathrm{WA} \\ 627 & 1 & & \mathrm{ug} / \mathrm{L} & 0 & \text { WA } \\ 610 & 1 & & \mathrm{ug} / \mathrm{L} & 0 & \mathrm{GE} \\ 620 & 1 & & \mathrm{ug} / \mathrm{L} & 0 & \mathrm{GE} \\ 630 & 1 & & \mathrm{ug} / \mathrm{L} & 0 & \mathrm{GE} \\ <.1 & 1 & \mathrm{UI} & \mathrm{pCi} / \mathrm{L} & 0 & \mathrm{TM} \\ <1.1 & 1 & \mathrm{UI} & \mathrm{pCi} / \mathrm{L} & 0 & \mathrm{TM} \\ 1.32 & 1 & & \mathrm{pCi} / \mathrm{L} & 0 & \mathrm{GP} \\ <.772 & 1 & \mathrm{UI} & \mathrm{pCi} / \mathrm{L} & 0 & \mathrm{GP} \\ 2900 & 1 & & \mathrm{pCi} / \mathrm{L} & 0 & \mathrm{GP} \\ 4.17 & 1 & & \mathrm{pCi} / \mathrm{mL} & 0 & \mathrm{TM} \\ 3.49 & 1 & & \mathrm{pCi} / \mathrm{mL} & 0 & \mathrm{TM} \\ 2990 & 1 & - & \mathrm{pCi} / \mathrm{L} & 0 & \mathrm{GP} \\ 124 & 1 & & \mathrm{ug} / \mathrm{L} & 2 & \mathrm{GE} \\ <87 & 1 & \mathrm{U} & \mathrm{ug} / \mathrm{L} & 0 & \mathrm{WA} \\ <87 & 1 & \mathrm{U} & \mathrm{ug} / \mathrm{L} & 0 & \mathrm{WA} \\ 144 & 1 & \mathrm{~J} & \mathrm{ug} / \mathrm{L} & 2 & \mathrm{GE} \\ 288 & 1 & & \mathrm{ug} / \mathrm{L} & 2 & \mathrm{GE} \\ <5 & 1 & \mathrm{U} & \mathrm{ug} / \mathrm{L} & 0 & \mathrm{GE} \\ <12 & 1 & \mathrm{U} & \mathrm{ug} / \mathrm{L} & 0 & \mathrm{WA} \\ <5 & 1 & \mathrm{U} & \mathrm{ug} / \mathrm{L} & 0 & \mathrm{GE} \\ <12 & 1 & \mathrm{U} & \mathrm{ug} / \mathrm{L} & 0 & \mathrm{WA} \\ 18.8 & 1 & & \mathrm{ug} / \mathrm{L} & 0 & \mathrm{GE} \\ 17.6 & 1 & & \mathrm{ug} / \mathrm{L} & 0 & \mathrm{WA} \\ 17.6 & 1 & & \mathrm{ug} / \mathrm{L} & 0 & \mathrm{WA} \\ 19 & 1 & & \mathrm{ug} / \mathrm{L} & 0 & \mathrm{GE} \\ 17.8 & 1 & & \mathrm{ug} / \mathrm{L} & 0 & \mathrm{GE} \\ & & & & & \end{array}$

* = exceeded holding time. $+=$ exceeded screening level or final primary drinking water standard. 
H ST Analyte

Cadmium, total recoverable Cadmium, total recoverable Cadmium, total recoverable Cadmium, total recoverable Cadmium, total recoverable Chromium, total recoverable Chromium, total recoverable Chromium, total recoverable Chromium, total recoverable Chromium, total recoverable Iron, total recoverable Iron, total recoverable Iron, total recoverable Iron, total recoverable Iron, total recoverable Lead, total recoverable Lead, total recoverable Lead, total recoverable Lead, total recoverable Lead, total recoverable Manganese, total recoverable Manganese, total recoverable Manganese,' total recoverable Manganese, total recoverable Manganese, total recoverable Mercury, total recoverable Mercury, total recoverable Mercury, total recoverable Mercury, total recoverable Nickel, total recoverable Radium, total alpha-emitting Radium, total alpha-emitting Radium-226

Radium-226

Radium-228

Radium-228

Selenium, total recoverable Selenium, total recoverable Selenium, total recoverable Selenium, total recoverable Silver, total recoverable Silver, total recoverable Silver, total recoverable Silver, total recoverable Silver, total recoverable Sodium, total recoverable Sodium, total recoverable Sodium, total recoverable Sodium, total recoverable Sodium, total recoverable

\begin{tabular}{|c|c|c|c|c|c|}
\hline Result & $\underline{\mathrm{DF}}$ & $\underline{\text { Mod }}$ & Unit & Flag & $\underline{\mathrm{Lab}}$ \\
\hline$<2$ & 1 & $\mathrm{U}$ & $\mathrm{ug} / \mathrm{L}$ & 0 & GE \\
\hline$<2$ & 1 & $\mathrm{U}$ & $\mathrm{ug} / \mathrm{L}$ & 0 & $\mathrm{GE}$ \\
\hline$<4.7$ & 1 & U & $\mathrm{ug} / \mathrm{L}$ & 0 & WA \\
\hline$<4.7$ & 1 & $U$ & $u g / L$ & 0 & WA \\
\hline$<2$ & 1 & U & $u g / L$ & 0 & $\mathrm{GE}$ \\
\hline$<4$ & 1 & $U$ & $\mathrm{ug} / \mathrm{L}$ & 0 & GE \\
\hline$<10$ & 1 & $U$ & ug/L & 0 & $\mathrm{w}_{1}$ \\
\hline$<4$ & 1 & $\mathrm{U}$ & $\mathrm{ug} / \mathrm{L}$ & 0 & \\
\hline$<4$ & 1 & $\mathrm{U}$ & $\mathrm{ug} / \mathrm{L}$ & 0 & \\
\hline$<10$ & 1 & U & ug/L & 0 & r \\
\hline 126 & 1 & & $\mathrm{ug} / \mathrm{L}$ & 0 & GE \\
\hline 122 & 1 & & ug/L & 0 & GE \\
\hline 127 & 1 & & $\mathrm{ug} / \mathrm{L}$ & 0 & $\mathrm{GE}$ \\
\hline 25.2 & 1 & $\mathbf{J}$ & ug/L & 0 & $N A$ \\
\hline 27.4 & 1 & & $\mathrm{ug} / \mathrm{L}$ & 0 & N \\
\hline 8.29 & 1 & & $\mathrm{ug} / \mathrm{L}$ & 0 & GE \\
\hline 3.89 & 1 & $\mathbf{J}$ & $\mathrm{ug} / \mathrm{L}$ & 0 & GE \\
\hline 13 & 1 & $\mathrm{~J}$ & ug/L & 0 & NA \\
\hline 5.47 & 1 & JV & $\mathbf{u g} / \mathrm{L}$ & 0 & GE \\
\hline$<13$ & 1 & $\mathrm{U}$ & $\mathrm{ug} / \mathrm{L}$ & 0 & NA \\
\hline 3.99 & 1 & & ug/L & 0 & $\mathrm{HE}$ \\
\hline 4.1 & 1 & $\mathrm{~J}$ & ug $/ \mathrm{L}$ & 0 & WA \\
\hline 3.8 & 1 & $\mathrm{~J}$ & ug/L & 0 & $\mathrm{VA}$ \\
\hline 3.92 & 1 & & $\mathrm{ug} / \mathrm{L}$ & 0 & GE \\
\hline 3.86 & 1 & & $u g / L$ & 0 & GE \\
\hline$<.2$ & 1 & U & ug/L & 0 & $\mathrm{GE}$ \\
\hline$<.67$ & 1 & U & $\mathbf{u g} / \mathrm{L}$ & 0 & WA \\
\hline$<.2$ & 1 & $\mathrm{U}$ & $\mathrm{ug} / \mathrm{L}$ & 0 & GE \\
\hline$<.67$ & 1 & $\mathrm{U}$ & $\mathrm{ug} / \mathrm{L}$ & 0 & W \\
\hline 5.4 & 1 & $\mathrm{~J}$ & $\mathrm{ug} / \mathrm{L}$ & 0 & GE \\
\hline$<-.2$ & 1 & UI & $\mathrm{pCi} / \mathrm{L}$ & 0 & GP \\
\hline$<0$ & 1 & UI & $\mathrm{pCi} / \mathrm{L}$ & 0 & GP \\
\hline$<.16$ & 1 & UI & $\mathrm{pCi} / \mathrm{L}$ & 0 & $\Gamma$ \\
\hline$<.17$ & 1 & UI & $\mathrm{pCi} / / \mathrm{L}$ & 0 & I \\
\hline$<1.3$ & 1 & UI & $\mathrm{pCi} / \mathrm{L}$ & 0 & TM \\
\hline 4.7 & 1 & V & $\mathrm{pCi} / \mathrm{L}$ & 0 & $I M$ \\
\hline$<5$ & 1 & $\mathrm{U}$ & ug/L & 0 & $\mathrm{GE}$ \\
\hline$<19$ & 1 & $U$ & ug/L & 0 & WA \\
\hline$<19$ & 1 & U & ug/L & 0 & WA \\
\hline$<5$ & 1 & $\mathrm{U}$ & $u g / L$ & 0 & GE \\
\hline$<.65$ & 1 & $U$ & $\mathrm{ug} / \mathrm{L}$ & 0 & GE \\
\hline .37 & 1 & JV & $\mathrm{ug} / \mathrm{L}$ & 0 & Wf \\
\hline$<2$ & 1 & $U$ & ug/L & 0 & W \\
\hline$<.65$ & 1 & $U$ & $\mathrm{ug} / \mathrm{L}$ & 0 & GE \\
\hline$<.65$ & 1 & $\mathrm{U}$ & $\mathrm{ug} / \mathrm{L}$ & 0 & GE \\
\hline 5330 & 1 & & $\mathrm{ug} / \mathrm{L}$ & 0 & GE \\
\hline 5580 & 1 & & $\mathrm{ug} / \mathrm{L}$ & 0 & GE \\
\hline 5180 & 1 & & $\mathrm{ug} / \mathrm{L}$ & 0 & \\
\hline 5130 & 1 & & $\mathrm{ug} / \mathrm{L}$ & 0 & \\
\hline 5620 & 1 & & $\mathrm{ug} / \mathrm{L}$ & 0 & \\
\hline
\end{tabular}

* = exceeded holding time. $+=$ exceeded screening level or final primary drinking water standard. 
H ST Analyte

$\begin{array}{ll} & \text { Sulfate } \\ & \text { Sulfate } \\ & \text { Sulfate } \\ & \text { Sulfate } \\ & \text { Sulfate } \\ * & \text { pH } \\ * & \text { pH } \\ * & \text { pH } \\ * & \text { pH }\end{array}$

Number of Records: 90
Result DF Mod Unit Flag Lab

$\begin{array}{llllll}1620 & 1 & & \mathrm{ug} / \mathrm{L} & 0 & \mathrm{GE} \\ 1730 & 1 & & \mathrm{ug} / \mathrm{L} & 0 & \text { WA } \\ 1680 & 1 & & \mathrm{ug} / \mathrm{L} & 0 & \text { WA } \\ 1690 & 1 & & \mathrm{ug} / \mathrm{L} & 0 & \text { WA } \\ 1570 & 1 & & \mathrm{ug} / \mathrm{L} & 0 & \mathrm{GE} \\ 5.56 & 1 & \mathrm{~J} & \mathrm{pH} & 0 & \mathrm{GE} \\ 5.52 & 1 & \mathrm{~J} & \mathrm{pH} & 0 & \text { WA } \\ 5.54 & 1 & \mathrm{~J} & \mathrm{pH} & 0 & \text { WA } \\ 5.59 & 1 & \mathrm{~J} & \mathrm{pH} & 0 & \mathrm{GE}\end{array}$

WELL: PAC2

SRS Coord. Lat/Longitude Screen Zone Elevation

N 43527.7 33.231299 Deg N E $66980.9-81.575029$ Deg W
Top of Casing Casing Pump Formation $284.8 \mathrm{ft} \mathrm{msl} 4^{\prime \prime}$ PVC $\mathrm{s}$ Water Table

\section{FIELD MEASUREMENTS}

Sample date: 04/20/95

Depth to water: $11.73 \mathrm{ft}(3.576 \mathrm{~m})$ below TOC

Water elevation: $273.07 \mathrm{ft}(83.25 \mathrm{~m}) \mathrm{msl}$

Sp. conductance: $74 \mathrm{uS} / \mathrm{cm}$

Turbidity: 2.7 NTU

Water evacuated before sampling: $279 \mathrm{gal}$
Time: 15:08

pH:6.3

Alkalinity: $17 \mathrm{mg} / \mathrm{L}$

Water temperature: $17.3 \mathrm{deg} C$

Volumes purged: 16.90 well volumes

\section{LABORATORY ANALYSES}

$\underline{\mathrm{H}} \underline{\mathrm{ST}}$ Analyte

Gross alpha

Nitrate as nitrogen

Nonvolatile beta

Tritium

Aluminum, total recoverable

Arsenic, total recoverable

Barium, total recoverable

Cadmium, total recoverable

Chromium, total recoverable

Iron, total recoverable

Lead, total recoverable

Manganese, total recoverable

Mercury, total recoverable

Radium-226

Radium-228

Radium-228

Radium-228
Result DF Mod " Unit Flag Lab

$\begin{array}{llllll}<-.3 & 1 & \text { UI } & \mathrm{pCi} / \mathrm{L} & 0 & \mathrm{TM}\end{array}$

$\begin{array}{llllll}33 & 1 & \mathrm{~J} & \mathrm{ug} / \mathrm{L} & 0 & \text { WA }\end{array}$

$\begin{array}{llllll}<1.4 & 1 & \text { UI } & \mathrm{pCi} / \mathrm{L} & 0 & \mathrm{TM}\end{array}$

$\begin{array}{llll}1.35 & 1 & \mathrm{pCi} / \mathrm{mL} & \mathrm{TM}\end{array}$

$\begin{array}{llllll}<8 & 1 & \mathrm{U} & \mathrm{ug} / \mathrm{L} & 0 & \text { WA }\end{array}$

$\begin{array}{llllll}3.5 & 1 & \text { JV } & \mathrm{ug} / \mathrm{L} & 0 & \text { WA }\end{array}$

$20.11 \quad$ ug/L 0 WA

$<4.7 \quad 1 \quad$ U $\quad$ ug/L $\quad 0 \quad$ WA

$\begin{array}{llllll}1.5 & 1 & \mathrm{~J} & \mathrm{ug} / \mathrm{L} & 0 & \text { WA }\end{array}$

$\begin{array}{lllll}5440 & 1 & \text { ug/L } & 2 & \text {.WA }\end{array}$

$<13 \quad 1 \quad$ U $\quad$ ug $/ \mathrm{L} \quad 0 \quad$ WA

61.611 ug/L 2 WA

$<.67 \quad 1 \quad$ U $\quad$ ug/L $\quad 0 \quad$ WA

$<.07 \quad 1 \quad$ UI $\mathrm{pCi} / \mathrm{L} \quad 0 \quad \mathrm{TM}$

$\begin{array}{llllll}63.7 & 1 & \mathrm{~J} & \mathrm{pCi} / \mathrm{L} & 2 & \mathrm{TM}\end{array}$

$<.9 \quad 1 \quad$ UI $\quad \mathrm{pCi} / \mathrm{L} \quad 0 \quad$ TM

$<.51$, UI $\quad \mathrm{pCi} / 20 \quad$ TM

* = exceeded holding time. $+=$ exceeded screening level or final primary drinking water standard. 
H ST Analyte

Selènium, total recoverable

Silver, total recoverable

Sodium, total recoverable

Sulfate

* $\quad \mathrm{pH}$

Number of Records: 22
Result DF Mod Unit Flag Lab

$\begin{array}{llllll}<19 & 1 & \mathrm{U} & \mathrm{ug} / \mathrm{L} & 0 & \text { WA } \\ 1.8 & 1 & \mathrm{~V} & \mathrm{ug} / \mathrm{L} & 0 & \text { WA } \\ 4030 & 1 & & \mathrm{ug} / \mathrm{L} & 0 & \text { WA } \\ 6770 & 1 & & \mathrm{ug} / \mathrm{L} & 0 & \text { WA } \\ 6.38 & 1 & \mathrm{~J} & \mathrm{pH} & 0 & \text { WA }\end{array}$

WELL: PAC3

SRS Coord. Lat/Longitude

N 43585.6 33.231232 Deg N

E 66861.4 -81.575456 Deg W
Screen Zone Elevation 282.9-252.9 ft msl
Top of Casing $289.9 \mathrm{ft} \mathrm{msl}$
Casing Pump 4" PVC S
Formation Water Table

\section{FIELD MEASUREMENTS}

Sample date: 04/20/95

Depth to water: $16.7 \mathrm{ft}(5.091 \mathrm{~m})$ below TOC Water elevation: $273.2 \mathrm{ft}(83.29 \mathrm{~m}) \mathrm{msl}$

Sp. conductance: $264 \mathrm{uS} / \mathrm{cm}$

Turbidity: 3.4 NTU

Water evacuated before sampling:64 gal
Time: $14: 23$

pH:5.5

Alkalinity: $11 \mathrm{mg} / \mathrm{L}$

Water temperature: $20 \mathrm{deg} C$

Volumes purged: 4.806 well volumes

\section{LABORATORY ANALYSES}

H $\quad \underline{\text { ST Analyte }}$

Gross alpha

Nitrate as nitrogen

Nonvolatile beta

Tritium

Aluminum, total recoverable

Arsenic, total recoverable

Barium, total recoverable

Cadmium, totál recoverable

Chromium, total recoverable

Iron, total recoverable

Lead, total recoverable

Manganese, total recoverable

Mercury, total recoverable

Radium-226

Radium-228

Selenium, total recoverable

Silver, total recoverable

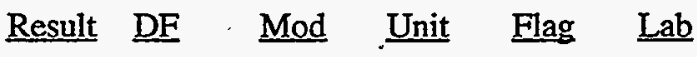

$\begin{array}{lllllll}<-4 & 1 & \text { UI } & \mathrm{pCi} / \mathrm{L} & 0 & \mathrm{TM}\end{array}$

$\begin{array}{lllll}589 & 1 & \text { ug/L } & 0 & \text { WA }\end{array}$

$\begin{array}{llllll}1.9 & 1 & \text { UI } & \mathrm{pCj} / \mathrm{L} & 0 & \mathrm{TM}\end{array}$

$\begin{array}{llll}5.85 & 1 & \mathrm{pCi} / \mathrm{mL} & \mathrm{TM}\end{array}$

$\begin{array}{llllll}23.6 & 1 & \mathrm{~J} & \mathrm{ug} / \mathrm{L} & 0 & \mathrm{WA}\end{array}$

$\begin{array}{llllll}<12 & 1 & \mathrm{U} & \text { ug } / \mathrm{L} & 0 & \text { WA }\end{array}$

$\begin{array}{llllll}69.6 & 1 & \mathrm{~J} & \mathrm{ug} / \mathrm{L} & 0 & \text { WA }\end{array}$

$<4.7 \quad 1 \quad$ U $\quad$ ug/L $\quad 0 \quad$ WA

$\begin{array}{llllll}3.8 & 1 & \mathrm{~J} & \mathrm{ug} / \mathrm{L} & 0 & \text { WA }\end{array}$

$\begin{array}{lllll}1480 & 1 & \text { ug/L } & 2 & \text { WA }\end{array}$

$\begin{array}{llllll}4.1 & 1 . & \mathrm{J} & \mathrm{ug} / \mathrm{L} & 0 & \text { WA }\end{array}$

$\begin{array}{lllll}16.2 & 1 & \mathrm{ug} / \mathrm{L} & 0 & \text { WA }\end{array}$

$\begin{array}{llllll}.67 & 1 & \mathrm{U} & \mathrm{ug} / \mathrm{L} & 0 & \text { WA }\end{array}$

$\begin{array}{llllll}.7 & 1 & \mathrm{~J} & \mathrm{pCi} / \mathrm{L} & 0 & \mathrm{TM}\end{array}$

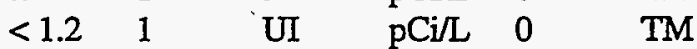

$<\begin{array}{llllll}19 & 1 & \mathrm{U} & \mathrm{ug} / \mathrm{L} & 0 & \text { WA }\end{array}$

$\begin{array}{llllll}.2 & 1 & \text { JV } & \text { ug/L } & 0 & \text { WA }\end{array}$

* = exceeded holding time. $+=$ exceeded screening level or final primary drinking water standard. 
H ST Analyte

Sodium, total recoverable

Sulfate

* $\quad \mathrm{pH}$

Number of Records: 20

\begin{tabular}{|c|c|c|c|c|}
\hline Result & $\underline{\mathrm{DF}}$ & $\underline{\text { Mod }}$ & Unit & Flag \\
\hline 330 & 1 & & $\mathrm{ug} / \mathrm{L}$ & 0 \\
\hline 72700 & 20 & & ug $/ \mathrm{L}$ & 0 \\
\hline 5.72 & & $J$ & $\mathrm{pH}$ & 0 \\
\hline
\end{tabular}

\section{WELL: PAC4}

SRS Coord. Lat/Longitude

N 43495.4 33.231036 Deg N

E $66863.2-81.575276$ Deg W

Screen Zone Elevation 280.6-250.6 ft msl

Top of Casing. $291.6 \mathrm{ft} \mathrm{msl}$
Casing. Pump 4" PVC S
Formation Water Table

\section{FIELD MEASUREMENTS}

Sample date: 04/21/95

Depth to water:6.31 ft (1.924 m) below TOC

Water elevation: $285.29 \mathrm{ft}(86.98 \mathrm{~m}) \mathrm{msl}$

Sp. conductance: $78 \mathrm{uS} / \mathrm{cm}$

Turbidity: 3.2 NTU

Water evacuated before sampling: 133 gal
Time: 10:15

pH:5.6

Alkalinity:2 $\mathrm{mg} / \mathrm{L}$

Water temperature: $19.4 \mathrm{deg} \mathrm{C}$

Volumes purged: 5.844 well volumes

\section{LABORATORY ANALYSES}

H ST Analyte

Gross alpha

Gross alpha

Gross alpha

Gross alpha

Nitrate as nitrogen

Nitrate as nitrogen

Nitrate-nitrite as nitrogen

Nitrate-nitrite as nitrogen

Nonvolatile beta

Nonvolatile beta

Nonvolatile beta

Nonvolatile beta

Tritium

Tritium

Tritium

Tritium

Aluminum, total recoverable

Aluminum, total recoverable

Aluminum, total recoverable

Aluminum, total recoverable

Arsenic, total recoverable

Arsenic, total recoverable

Arsenic, total recoverable
Result DF Mod Unit $\underline{\text { Flag }} \underline{\underline{\text { Lab }}}$

$\begin{array}{llllll}277 & 1 & \text { UII } & \mathrm{pCi} / \mathrm{L} & 0 & \mathrm{GP}\end{array}$

$<.575 \cdot 1$ UJ $\mathrm{pCi} / \mathrm{L} \quad 0 \quad \mathrm{GP}$

$\begin{array}{llllll}1 & 1 & \mathrm{~J} & \mathrm{pCi} / \mathrm{L} & 0 & \mathrm{TM}\end{array}$

$\begin{array}{llllll}<.1 & 1 & \text { UI } & \mathrm{pCi} / \mathrm{L} & 0 & \mathrm{TM}\end{array}$

$\begin{array}{llllll}709 & 1 & \text { ug/L } & 0 & \text { WA }\end{array}$

$\begin{array}{lllll}712 & 1 & \text { ug/L } & 0 & \text { WA }\end{array}$

$\begin{array}{lllll}830 & 1 & \mathrm{ug} / \mathrm{L} & 0 & \mathrm{GE}\end{array}$

$800 \quad 1 \quad$ ug $/ \mathrm{L} \quad 0 \quad \mathrm{GE}$

$<.8 \quad 1$. UI $\mathrm{pCi} / \mathrm{L} \quad 0 \quad \mathrm{TM}$

$<.375 \quad 1 \quad$ UI $\quad \mathrm{pCi} / \mathrm{L} \quad 0 \quad$ GP

$\begin{array}{llllll}<1.1 & 1 & \text { UI } & \mathrm{pCi} / \mathrm{L} & 0 & \mathrm{TM}\end{array}$

$<.469 \quad 1 \quad$ UI $\quad \mathrm{pCi} / \mathrm{L} \quad 0 \quad$ GP

$\begin{array}{lllll}2410 & 1 & \mathrm{pCi} / \mathrm{L} & 0 & \mathrm{GP}\end{array}$

$\begin{array}{llll}2.37 & 1 & \mathrm{pCi} / \mathrm{mL} & 0\end{array}$

$\begin{array}{lllll}2850 & 1 & \mathrm{pCi} / \mathrm{L} & 0 & \mathrm{GP}\end{array}$

$\begin{array}{llll}2.64 & 1 & \mathrm{pCi} / \mathrm{mL} & \mathrm{TM}\end{array}$

$\begin{array}{lllll}126 & 1 & \mathrm{ug} / \mathrm{L} & 2 & \mathrm{GE}\end{array}$

$\begin{array}{lllll}158 & 1 & \mathrm{ug} / \mathrm{L} & 2 & \mathrm{GE}\end{array}$

$\begin{array}{llllll}<87 & 1 & \text { U } & \text { ug/L } & 0 & \text { WA }\end{array}$

$<87 \quad 1 \quad$ U $\quad$ ug/L $\quad 0 \quad$ WA

$\begin{array}{llllll}<5 & 1 & \mathrm{U} & \mathrm{ug} / \mathrm{L} & 0 & \mathrm{GE}\end{array}$

$\begin{array}{llllll}<5 & 1 & \mathrm{U} & \mathrm{ug} / \mathrm{L} & 0 & \mathrm{GE}\end{array}$

$\begin{array}{llllll}2 & 1 & \mathrm{~J} & \mathrm{ug} / \mathrm{L} & 0 & \text { WA }\end{array}$

* $=$ exceeded holding time. $+=$ exceeded screening level or final primary drinking water standard. 


\section{$\underline{H} \underline{\text { ST }}$ Analyte}

Arsenic, total recoverable Arsenic, total recoverable Barium, total recoverable Barium, total recoverable Barium, total recoverable Barium, total recoverable Cadmium, total recoverable Cadmium, total recoverable Cadmium, total recoverable Cadmium, total recoverable Chromium, total recoverable Chromium, total recoverable Chromium, total recoverable Chromium, total recoverable Iron, total recoverable Iron, total recoverable Iron, total recoverable Iron, total recoverable Lead, total recoverable Lead, total recoverable Lead, total recoverable . Lead, total recoverable Lead, total recoverable Manganese, total recoverable Manganese, total recoverable Manganese, total recoverable Manganese, total recoverable Mercury, total recoverable Mercury, total recoverable Mercury, total recoverable Mercury, total recoverable Mercury, total recoverable Radium, total alpha-emitting Radium, total alpha-emitting Radium-226

Radium-226

Radiùm-228

Radium-228

Selenium, total recoverable Selenium, total recoverable Selenium, total recoverable Selenium, total recoverable Selenium, total recoverable Silver, total recoverable Silver, total recoverable Silver, total recoverable Silver, total recoverable Sodium, total recoverable Sodium, total recoverable Sodium, total recoverable
Result DF Mod Unit Fiag Lab

$<5,1 \quad$ U $\quad \begin{array}{llll}\text { ug/L } & 0 & \text { GE }\end{array}$

12.71 ug/L 0 GE

$\begin{array}{lllll}11.8 & 1 & \text { ug/L } & 0 & \text { WA }\end{array}$

$\begin{array}{lllll}11.9 & 1 & \text { ug/L } & 0 & \text { WA }\end{array}$

$\begin{array}{lllll}12.6 & 1 & \mathrm{ug} / \mathrm{L} & 0 & \mathrm{GE}\end{array}$

$\begin{array}{llllll}2 & 1 & U & u g / L & 0 & G E\end{array}$

$<4.7 \quad 1 \quad$ U $\quad$ ug/L $\quad 0 \quad$ WA

$<2 \quad 1 \quad \mathrm{U} \quad \mathrm{ug} / \mathrm{L}{ }^{\circ} 0, \mathrm{GE}$

$<4.7,1 \quad \mathrm{U}, \mathrm{ug} / \mathrm{L} \quad 0 \quad$ WA

$<4 \quad 1 \quad$ U $\quad . \quad u g / L \quad 0 \quad$ GE

$\begin{array}{lllll}5.17 & 1 & \mathrm{ug} / \mathrm{L} & 0 & \mathrm{GE}\end{array}$

$\begin{array}{llllll}<10 & 1 & \mathrm{U} & . \mathrm{ug} / \mathrm{L} & 0 & \text { WA }\end{array}$

$<10 \quad 1 \quad \mathrm{U}, \mathrm{ug} / \mathrm{L} \quad 0 \quad$ WA

$61.21 \quad$ ug/L 0 GE

$\begin{array}{llllll}20.8 & 1 & \mathrm{~J} & \mathrm{ug} / \mathrm{L} & 0 & \text { WA }\end{array}$

3511 ug/L 0 WA

$\begin{array}{lllll}51.3 & 1 & \mathrm{ug} / \mathrm{L} & 0 & \mathrm{GE}\end{array}$

$\begin{array}{llllll}.87 & 1 & \mathrm{JV} & \mathrm{ug} / \mathrm{L} & 0 & \mathrm{GE}\end{array}$

$\begin{array}{lllllll}.88 & 1 & \text { JV } & \text { ug/L } & 0 & \text { GE }\end{array}$

$<\begin{array}{llllll}13 & 1 & \mathrm{U} & \mathrm{ug} / \mathrm{L} & 0 & \text { WA }\end{array}$

$\begin{array}{llllll}13 & 1 & \mathrm{U} & \mathrm{ug} / \mathrm{L} & 0 & \text { WA }\end{array}$

$\begin{array}{llllll}1.1 & 1 & \text { JV } & \mathrm{ug} / \mathrm{L} & 0 & \mathrm{GE}\end{array}$

$\begin{array}{lllll}2.6 & 1 & \mathrm{ug} / \mathrm{L} & 0 & \mathrm{GE}\end{array}$

$\begin{array}{llllll}<.7 & 1 & \mathrm{U} & \mathrm{ug} / \mathrm{L} & 0 & \text { WA }\end{array}$

$<5.7 \quad 1 \quad$ U $\quad$ ug/L 0 WA

$\begin{array}{lllll}2.73 & 1 & \mathrm{ug} / \mathrm{L} & 0 & \mathrm{GE}\end{array}$

$<.2 \quad 1 \quad$ U $\quad$ ug/L $0 . \quad$ GE

$<.21 \quad$ U $\quad$ ug/L $0 \quad$ GE

$<.67 \quad 1 \quad \cdot \quad \mathrm{U} \quad \mathrm{ug} / \mathrm{L} \quad 0 \quad$ WA

$\begin{array}{llllll}.041 & 1 & \mathrm{~J} & \mathrm{ug} / \mathrm{L} & 0 & \mathrm{GE}\end{array}$

$<.67 \quad 1 \quad$ U $\quad$ ug $/ \mathrm{L} \quad 0 \quad$ WA

$<0 \quad 1 \quad$ UI $\quad \mathrm{pCi} / \mathrm{L} \quad 0 \quad$ GP

$<-.3 \quad 1 \quad$ UI $\quad \mathrm{pCi} / \mathrm{L} \quad 0 \quad$ GP

$\begin{array}{llllll}.21 & 1 & \mathrm{~J} & \mathrm{pCi} / \mathrm{L} & 0 & \mathrm{TM}\end{array}$

$\begin{array}{llllll}.18 & 1 & \mathrm{~J} & \mathrm{pCi} / \mathrm{L} & 0 & \mathrm{TM}\end{array}$

$<.5 \quad 1 \quad$ UI $\quad \mathrm{pCi} / \mathrm{L} \quad 0 \quad 0 \quad \mathrm{TM}$

$<.61$. UI $\mathrm{pCi} / \mathrm{L} 0 . \quad \mathrm{TM}$

$\begin{array}{llllll}1.29 & 1 & \mathrm{~J} & \mathrm{ug} / \mathrm{L} & 0 & \mathrm{GE}\end{array}$

$<19 \quad 1 . \quad \mathrm{U} \quad \mathrm{ug} / \mathrm{L} \quad 0 \quad \mathrm{WA}$

$\begin{array}{llllll}19 & 1 & \mathrm{U} & \mathrm{ug} / \mathrm{L} & 0 & \text { WA }\end{array}$

$\begin{array}{llllll}<5 & 1 & \mathrm{U} & \mathrm{ug} / \mathrm{L} & 0 & \mathrm{GE}\end{array}$

$\begin{array}{llllll}1.69 & 1 & \mathrm{~J} & \mathrm{ug} / \mathrm{L} & 0 & \mathrm{GE}\end{array}$

$<\begin{array}{llllll}.65 & 1 & \mathrm{U} & \mathrm{ug} / \mathrm{L} & 0 & \mathrm{GE}\end{array}$

$<.6511 \quad \mathrm{U} \quad \mathrm{ug} / \mathrm{L} .00 \mathrm{GE}$

$<1.41 \quad$ U $\quad$ ug/L 00 WA

$\begin{array}{llllll}1.4 & 1 & \text { U } & \mathrm{ug} / \mathrm{L} & 0 & \text { WA }\end{array}$

$1280011 \quad$ ug/L $\quad 0 \quad$ GE

$\begin{array}{lllll}12900 & 1 & \mathrm{ug} / \mathrm{L} & 0 & \mathrm{GE}\end{array}$

$\begin{array}{lllll}11900 & 1 & \text { ug/L } & 0 & \text { WA }\end{array}$

* = exceeded holding time. $+=$ exceeded screening level or final primary drinking water standard. 
H ST Analyte

\begin{tabular}{ll} 
& \multicolumn{2}{l}{ Sodium, total recoverable } \\
& Sulfate \\
& Sulfate \\
& . \\
& Sulfate \\
$*$ & Sulfate \\
$*$ & pH \\
$*$ & pH \\
$*$ & pH \\
Number of Records: 82
\end{tabular}

\begin{tabular}{|c|c|c|c|c|}
\hline Result & $\underline{\mathrm{DF}}$ & Mod & Unit & Flag \\
\hline 11800 & 1 & & $u g / L$ & 0 \\
\hline 20500 & 1 & & $\mathrm{ug} / \mathrm{L}$ & 0 \\
\hline 23400 & 10 & & ug $/ L$ & 0 \\
\hline 20300 & 1 & & $\mathrm{ug} / \mathrm{L}$ & 0 \\
\hline 22600 & 10 & & $\mathrm{ug} / \mathrm{L}$ & 0 \\
\hline 5.51 & 1 & J & $\mathrm{pH}$ & 0 \\
\hline 5.49 & 1 & $\mathbf{J}$ & $\mathrm{pH}$ & 0 \\
\hline 5.51 & 1 & $\mathrm{~J}$ & $\mathrm{pH}$ & 0 \\
\hline 5.51 & 1 & $\mathrm{~J}$ & $\mathrm{pH}$ & 0 \\
\hline
\end{tabular}

\section{WELL: PAC5}

SRS Coiord. Lat/Longitude N 43561.7 33.231254 Deg N E 66907.1 -81.575289 Deg W
Screen Zone Elevation 275.1-255.1 ft msl

Top of Casing $289.3 \mathrm{ft} \mathrm{msl}$
Casing Pump 4" PVC S
Formation Water Table

\section{FIELD MEASUREMENTS}

Sample date: 04/21/95

Depth to water: $12.46 \mathrm{ft}(3.799 \mathrm{~m})$ below T.OC

Water elevation: $276.84 \mathrm{ft}(84.40 \mathrm{~m}) \mathrm{msl}$

Sp. conductance:606 uS/cm

Turbidity:15.4 NTU

Water evacuated before sampling: $12 \mathrm{gal}$
Time: 09:40

$\mathrm{pH}: 7$

Alkalinity:222 mg/L

Water temperature: $17.8 \mathrm{deg} \mathrm{C}$

Volumes purged: .8414 well volumes

\section{LABORATORY ANALYSES}

H ST Analyte

Gross alpha

Nitrate as nitrogen

Nonvolatile beta

Tritium

Aluminum, total recoverable

Arsenic, total recoverable ,

Barium, total recoverable

Cadmium, total recoverable

Chromium, total recoverable

Iron, total recoverable

Lead, total recoverable

Manganese, total recoverable

Mercury, total recoverable

Radium-226

Radium-228

Selenium, total recoverable

Silver, total recoverable

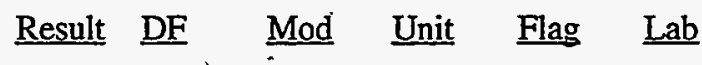

\begin{tabular}{|c|c|c|c|c|c|}
\hline 3.1 & 1 & - & $\mathrm{pCi} / \mathrm{L}$ & 0 & $T M$ \\
\hline 116 & 1 & & ug/L & 0 & WA \\
\hline 6.4 & 1 & & $\mathrm{pCi} / \mathrm{L}$ & 0 & $\mathrm{TM}$ \\
\hline 2.19 & 1 & & $\mathrm{pCi} / \mathrm{mL}$ & 0 & $\mathrm{TM}$ \\
\hline 278 & 1 & & ug/L & 2 & WA \\
\hline$<12$ & 1 & $\mathrm{U}$ & $\mathrm{ug} / \mathrm{L}$ & 0 & WA \\
\hline 102 & 1 & & $\mathrm{ug} / \mathrm{L}$ & 0 & WA \\
\hline$<4.7$ & 1 & U & $\mathrm{ug} / \mathrm{L}$ & 0 & WA \\
\hline$<10$ & 1 & $\mathrm{U}$ & $\mathrm{ug} / \mathrm{L}$ & 0 & WA \\
\hline 761 & 1 & & $\mathrm{ug} / \mathrm{L}$ & 2 & WA \\
\hline 1.8 & 1 & $\mathrm{~J}$ & $\mathrm{ug} / \mathrm{L}$ & 0 & WA \\
\hline 306 & 1 & & $\mathrm{ug} / \mathrm{L}$ & 2 & WA \\
\hline$<.67$ & 1 & $U$ & $\mathrm{ug} / \mathrm{L}$ & 0 & WA \\
\hline .42 & 1 & & $\mathrm{pCi} / \mathrm{L}$ & 0 & TM \\
\hline 2.1 & 1 & & $\mathrm{p} \mathrm{Ci} / \mathrm{L}$ & 0 & TM \\
\hline$<19$ & 1 & U & $\mathrm{ug} / \mathrm{L}$ & 0 & WA \\
\hline$<1.4$ & 1 & $\mathrm{U}$ & $\mathrm{ug} / \mathrm{L}$ & 0 & WA \\
\hline
\end{tabular}

* = exceeded holding time. $+=$ exceeded screening level or final primary drinking water standard. 
$\underline{\mathrm{H}} \quad \underline{\mathrm{ST}}$ Analyte

$\begin{array}{ll} & \text { Sodium, total recoverable } \\ & \text { Sulfate } \\ * & \mathrm{pH} \\ * & \mathrm{pH} \\ \text { Number of Records: } 21\end{array}$

Result DF Mod Unit Flag Lab

$41700 \quad 1$

$51100 \quad 20$

$\begin{array}{ll}7.05 & .1\end{array}$

$7.06 \quad 1$ $\mathrm{ug} / \mathrm{L} \quad 0 \quad$ WA

ug/L $0 \quad$ WA

$\mathrm{pH} \quad 0 \quad$ WA

$\mathrm{pH} \quad 0 \quad$ WA

\section{WELL: PAC6}

SRS Coord. Lat/Longitude

N 43580.1 33.231274 Deg N

E $66894.7-81.575358$ Deg W
Screen Zone Elevation . Top of Casing 275,2-255.2 ft.msl
Casing Pump 4" PVC S
Formation Water Table

\section{FIELD MEASUREMENTS}

Sample date: 04/21/95

Depth to water: $12.72 \mathrm{ft}$ ( $3.878 \mathrm{~m}$ ) below TOC

Water elevation: $276.68 \mathrm{ft}(84.35 \mathrm{~m}) \mathrm{ms}$

Sp. conductance:206 uS/cm

Turbidity:10.9 NTU

Water evacuated before sampling: $13 \mathrm{gal}$

Well went dry during purging; samples collected after well recovered

\section{LABORATORY ANALYSES}

H $\quad \underline{\text { ST }}$ Analyte

Gross alpha

Nitrate as nitrogen

Nonvolatile beta

Tritium

Aluminum, total recoverable

Arsenic, total recoverable

Barium, total recoverable

Cadmium, total recoverable

Chromium, total recoverable

Iron, total recoverable

'Lead, total recoverable

Manganese, total recoverable

Mercury, total recoverable

Radium-226

Radium-228

Selenium, total recoverable

Silver, total recoverable

Sodium, total recoverable

* $\quad \mathrm{pH}$

Sulfate

Number of Records: 20
Time: 09:28

pH:6.4

Alkalinity: $54 \mathrm{mg} / \mathrm{L}$

Water temperature: $17.8 \mathrm{deg} C$

Volumes purged: .9226 well volumes 


\section{- Appendix E}

\section{Data Quality/Usability Assessment}


THIS PAGE LEFT BLANK INTENTIONALLY. 


\section{Data Quality/Usability Assessment}

Quality assurance/quality control $(\mathrm{OA} / \mathrm{QC})$ procedures relating to accuracy and precision of analyses performed on groundwater samples are followed in the field and laboratory and are reviewed prior to publication of results. The review by the Environmental Protection Department/Environmental Monitoring Section (EPD/EMS) of the volume of analytical data acquired each quarter and presented in various reports is an ongoing process; its review of the QA/OC data cannot be completed in time to meet the deadlines for the reports required by the Resource Conservation and Recovery Act and associated regulations. Other site and regulatory personnel can obtain further information on the data quality and usability in a variety of ways, including those described below.

\section{Data Qualification}

The contract laboratories continually assess their own accuracy and precision according to U.S. Environmental Protection Agency (EPA) guidelines. They submit sample- or batch-specific $\mathrm{QA} / \mathrm{OC}$ information either at the same time as analytical results or in quarterly summaries. Properly defined and used result modifiers (also referred to as qualifiers) can be a key component in assessing data usability. Result modifiers designed by EPD/EMS and used by the primary laboratories are presented in Appendix D.

\section{Assessment of Accuracy of the Data}

Accuracy, or the nearness of the reported result to the true concentration of a constituent in a sample, can be assessed in several ways.

A laboratory's general accuracy can be judged by analysis of results obtained from known samples. The non-radionuclide contract laboratories analyze commercial reference samples every quarter at EPD/EMS' request. The results of these analyses are presented in the EPD/EMS groundwater monitoring quarterly reports. The primary laboratories also seek or maintain state certification by participating periodically in performance studies; reference samples and analysis of results are provided by EPA. Results of these studies also are published in the EPD/EMS. quarterly reports.

Analysis of blanks provides a tool for assessing the accuracy of both sampling and laboratory analysis. Results for all field blanks for the quarter can be found in the EPD/EMS quarterly reports. Any field or laboratory blanks that exceed established minimums are identified in the same reports, in tables associating them with groundwater samples analyzed in the same batches.

Surrogates, organic compounds similar in chemical behavior to the compounds of interest but not normally found in environmental samples, are used to monitor the effect of the matrix on the accuracy of analyses for organic parameters. For example, for analyses of volatile organics be EPA Method 8240, three surrogate compounds are added to all samples and blanks in each analytical batch. In analyses of semivolatile organics, three acid compounds and three base/neutral compounds are used. Two surrogates are used in organochlorine pesticides analyses. Percent recoveries for surrogate analyses are calculated by laboratory personnel, reported to EPD/EMS, reviewed, and entered into the database, but they are not published. If recoveries are not within specified limits, the laboratory is 
expected to reanalyze the samples or attach qualifiers to the data identifying the ariomalous results.

Sample-specific accuracy for both organic and inorganic parameters can be assessed by examination of matrix spike/matrix spike duplicate results. A sample is analyzed unspiked to determine a baseline set of values. A second portion of the sample is spiked with known concentrations of compounds appropriate to the analyses being performed, typically five volatile organic compounds for volatile organics analyses, eleven semivolatile compounds for semivolatiles, six pesticide compounds for pesticides, all metals for metals analyses by SW-846 methods (EPA, 1986), and a known quantity of cyanide for cyanide analysis. The percentage of . the spike compound that is recovered (i.e., measured in excess of the value obtained for the unspiked sample) is a direct measure of analytical accuracy. EPA requires matrix spike/matrix spike duplicates to be run at least once per 20 samples of similar matrix.

Matrix spike/matrix spike duplicate results are reported to EPD/EMS but are not published. For organic compounds, according to EPA guidelines, no action is taken on the basis of matrix spike/matrix spike duplicate data alone (i.e., no result modifiers are assigned solely on the basis of matrix spike results); however, the results can indicate if a laboratory is having a systematic problem in the analysis of one or more analytes.

In the case of inorganic compounds, such as metals, the matrix spike sample analysis provides information about the effect of each sample matrix on the digestion and measurement methodology. Data qualifiers assigned by the laboratories on the basis of the percentage of spike recovery are reported in the published results tables.

\section{Assessment of Precision}

Precision of the analyses, or. agreement of a set of replicate results among themselves, is assessed through the use of duplicates initiated by the laboratory and blind replicates provided by EPD/EMS. The results of duplicate and replicate analyses are presented in those results tables of the quarterly reports which report only one quarter of data, usually during first, second, and third quarters. Duplicate and replicate results are not presented in results tables that report more than one quarter of data, generally provided in fourth quarter reports. In this case, the results tables instead present only the highest result for each analyte for each quarter of the year.

The laboratories assess precision by calculating the relative percent difference (RPD) for each pair of laboratory-initiated duplicate results. One of the contract laboratories uses a data qualifier(J3) to modify metals analyses when the RPD for laboratory duplicates is greater than 20 percent.

Additional statistical comparisons of laboratory duplicate and blind replicate results, both intra- and interlaboratory, are presented in the EPD/EMS quarterly reports. The calculation used for these reports is the mean relative difference (MRD) which is similar to EPA's RPD except that the MRD is the average of all the RPD values from one laboratory for each compound (intralaboratory MRD) or all the RPD values from all laboratories for each compound (interlaboratory MRD), during one quarter. Because detection limits may vary among samples, the MRD requires calculation of a reference detection limit, which is the detection limit at the 90 th percentile of the 
array of limits in the population of all duplicate and replicate analyses for a given analyte during a particular quarter. The MRD is not method-specific.

\section{Method-Specific Accuracy and Precision}

The contract laboratories' EPA-approved laboratory procedures include OA/OC requirements as an integral part of the methods. Thus, knowledge of the method used in obtaining data is an important component of determining data usability. EPA has conducted extensive research and development on the methods approved for the analysis of water and waste water; information on the accuracy and precision of a method is available from EPA publications, as is full information on required $\mathrm{OA} / \mathrm{OC}$ procedures. A listing of the methods used by the primary laboratories during fourth quarter 1993 is given below along with the source for the method description. Many, if not all, of these sources include presentations of representative accuracy and precision results.

\section{Methods Used by the Contract Laboratories}

\begin{tabular}{|c|c|c|c|}
\hline Method & \multicolumn{2}{|l|}{ Used to Analyze } & Şource \\
\hline EPA120.1 & \multirow{2}{*}{\multicolumn{2}{|c|}{ Specific conductance }} & EPA EMSL, 1983 \\
\hline EPA150.1 & & & EPA EMSL, 1983 \\
\hline EPA160.1 & \multicolumn{2}{|l|}{$\begin{array}{l}\text { pH } \\
\text { Total dissolved solids }\end{array}$} & EPA EMSL, 1983 \\
\hline EPA160.2 & \multicolumn{2}{|c|}{ Total dissolved solids, total suspended solids } & EPA.EMSL, 1983 \\
\hline EPA180.1 & \multicolumn{2}{|c|}{ Turbidity } & EPA EMSL, 1983 \\
\hline EPA200.7 & \multicolumn{2}{|l|}{ Metals } & EPA EMSL, 1983 \\
\hline EPA204.2 & & EPA EMSL, 1983 \\
\hline EPA206.2 & Arsenic & . & EPA EMSL, 1983 \\
\hline EPA239.2 & Lead & & EPA EMSL, 1983 \\
\hline EPA245.1 & Mercury & & EPA EMSL, 1983 \\
\hline EPA270.2 & Selenium & " & EPA EMSL, 1983 \\
\hline EPA279.2 & Thallium & • & EPA EMSL, 1983 \\
\hline EPA300.0 & Chloride, nitrite, sulfate & & EPA EMSL, 1991 \\
\hline EPA310.1 & Alkalinity & & EPA EMSL, 1983 \\
\hline EPA325.2 & Chloride & & EPA EMSL, 1983 \\
\hline EPA335.3 & Cyanide & . & EPA EMSL, 1983 \\
\hline EPA340.2 & Fluoride & & EPA EMSL, 1983 \\
\hline EPA353.1 & Nitrogen, nitrate-nitrite & & EPA EMSL, 1983 \\
\hline EPA353.2 & Nitrogen, nitrate, nitrite, or com & bined & EPA EMSL, 1983 \\
\hline EPA365.1 & Phosphorus, all forms (reported & as total phosphates) & EPA EMSL, 1983 \\
\hline EPA365.2 & Phosphorus, all forms (reported & as total phosphates) & EPA EMSL, 1983 \\
\hline EPA376.2 & Sulfide & & EPA EMSL, 1983 \\
\hline EPA413.1 & Oil \& grease & & EPA EMSL, 1983 \\
\hline EPA415.1 & $\begin{array}{l}\text { Dissolved organic carbon, total } \\
\text { carbon }\end{array}$ & norganic carbon, total organic & EPA EMSL, 1983 \\
\hline EPA418.1 & Total petroleum hydrocarbons & & EPA EMSL, 1983 \\
\hline EPA420.2 & Phenols & & EPA EMSL, 1983 \\
\hline EPA900.0 & Gross alpha, nonvolatile beta & & EPA EMSL, 1980 \\
\hline EPA900.1 & Total alpha-emitting radium & & EPA EMSL, 1980 \\
\hline EPA906.0 & Tritium & & EPA EMSL, 1980 \\
\hline EPA6010 & Metals & & EPA, 1986 \\
\hline EPA7041 & Antimony & & EPA, 1986 \\
\hline EPA7060 & Arsenic & & EPA, 1986 \\
\hline EPA7421 & Lead & & EPA, 1986 \\
\hline EPA7470 & Mercury & & EPA, 1986 \\
\hline EPA7740 & Selenium & & EPA, 1986 \\
\hline EPA7841 & Thallium & & EPA, 1.986 \\
\hline
\end{tabular}




$\begin{array}{llr}\text { EPA8010 } & \text { Chlorinated volatile organics } & \text { EPA, } 1986 \\ \text { EPA8080 } & \text { Organochlorine pesticides and PCBs } & \text { EPA, } 1986 \\ \text { EPA8150 } & \text { Chlorinated herbicides } & \text { EPA, } 1986 \\ \text { EPA8240 } & \text { GCMS volatiles } & \text { EPA, } 1986 \\ \text { EPA8270' } & \text { GCMS semivolatiles } & \text { EPA, } 1986 \\ \text { EPA8280 } & \text { Dioxins and furans } & \text { EPA, } 1986 \\ \text { EPA9012 } & \text { Cyanide } & \text { EPA, } 1986 \\ \text { EPA9020 } & \text { Total organic halogens } & \text { EPA, } 1986 \\ \text { EPA9020A } & \text { Total organic halogens } & \text { EPA, } 1986 \\ \text { EPA9030 } & \text { Sulfide } & \text { EPA, } 1986 \\ \text { EPA9060 } & \text { Dissolved organic carbon, total inorganic carbon, total organic } & \text { EPA, } 1986\end{array}$

An example of available method-specific $Q A / O C$ information is that for the analysis of metals by EPA Method 6010/200.7 (EPA, 1986/EPA EMSL, 1983). The primary laboratories, General Engineering Laboratories (GE) and Roy F. Weston, inc. (Weston), use this inductively coupled plasma (ICP) atomic.emission spectrometric method.

The following precision and accuracy data are based on the experience of seven laboratories that applied the ICP technique to acid-distilled water matrices that had been spiked with various metal concentrates. (Note: Not all seven laboratories analyzed all 14 elements.) The references give results for samples having three concentration ranges; the results here are for samples having the lowest values, similar to actual groundwater results for SRS.

ICP Precision and Accuracy Data

\begin{tabular}{|c|c|c|c|}
\hline Element & True value (ma/L) & $\begin{array}{l}\text { Mean reported } \\
\text { value (mg/L) }\end{array}$ & \\
\hline Aluminum & 60 & 62 & \\
\hline Arsenic & 22 & 19 & \\
\hline Beryllium & 20 & 20 & \\
\hline Cadmium & 2.5 & 2.9 & \\
\hline Chromium & 10 & 10 & \\
\hline Cobalt & 20 & 20 & \\
\hline Copper & 11 & 11 & \\
\hline Iron & 20 & 19 & \\
\hline Lead . & 24 & 30 & \\
\hline Manganese & 15 & 15 & \\
\hline Nickel . & 30 & 28 & \\
\hline Selenium & 6 & 8.5 & \\
\hline Vanadium & 70 & 69 & \\
\hline Zinc & 16 & 19 & \\
\hline
\end{tabular}

Mean percent $\underline{\text { RSD }}^{\mathbf{2}}$

33

23

9.8

16

4.1

40

15

32

6.7

11

42

2.9

45

a Relative standard deviation. In EPA (1986), the column heading is Mean Standard Deviation (\%). .

As another example, EPA Method 601/8010 (EPA, 1991/EPA, 1986) is used by both GE and Weston for analyses of halogenated volatile organics. In the presentation of the method in both references, the following table gives methodspecific accuracy and precision as functions of concentration. Contract laboratories are expected to achieve or at least approach these limits. 
Accuracy and Precision as Functions of Concentration for EPA Method 601/8010

Parameter

Bromodichloromethane

Bromoform

Bromomethane

Carbon tetrachloride

Chlorobenzene

Chloroethane

2-Chloroethyl vinyl etherf

Chloroform

Chloromethane

Dibromochloromethane

1,2-Dichlorobenzene

1,3-Dichlorobenzene

1,4-Dichlorobenzene

1,1-Dichloroethane

1,2-Dichloroethane

1,1-Dichloroethene

trans-1,2-Dichloroethene

Dichloromethane

(Methylene chloride)

1,2-Dichloropropanef cis-1,3-Dichloropropenef trans-1,3-Dichloropropenef

1,1,2,2-Tetrachloroethane

Tetrachloroethylene

1,1,1-Trichloroethane

1,1,2-Trichloroethane

Trichloroethylene

Trichlorofluoromethane

Vinyl chloride

Accuracy as
recovery, $X^{\prime}-(\mathrm{mg} / \mathrm{L})$

Single analyst

${\text { precision }\left(\mathrm{mg} / \mathrm{L}^{\mathrm{b}}\right.}^{\mathrm{b}}$

Overall

$1.12 C-1.02^{c}$

$0.96 C-2.05$

$0.76 C-1.27$

$0.98 C-1.04$

$1.00 C-1.23$

$0.99 C-1.53$

$1.00 \mathrm{C}$

$0.93 C-0.39$

$0.77 C+0.18$

$0.94 C+2.72$

$0.93 C+1.70$

$0.95 C+0.43$

$0.93 C-0.09$

$0.95 C-1.08$

$1.04 C-1.06$

$0.98 C-0.87$

$0.97 C-0.16$

$0.91 C-0.93$

$1.00 \mathrm{C}$

$1.00 \mathrm{C}$

$1.00 \mathrm{C}$

$0.95 C+0.19$

$0.94 C+0.06$

$0.90 C-0.16$

$0.86 C+0.30$

$0.87 C+0.48$

$0.89 C-0.07$

$0.97 C-0.36$ $0.11+0.04^{\circ}$

$0.12+0.58$

$0.28+0.27$

$0.15+0.38$

$0.15-0.02$

0.14-0.13

0.20

$0.13+0.15$

$0.28-0.31$

$0.11+1.10$

$0.20+0.97$

$0.14+2.33$

$0.15+0.29$

$0.09+0.17$

$0.11+0.70$

$0.21-0.23$

$0.11+1.46$

$0.11+0.33$

0.13

0.18

0.18 .

$0.14+2.41$.

$0.14+0.38$

$0.15+0.04$

0.13-0.14

$0.13-0.03$

$0.15+0.67$
$0.13+0.65$ precision (ma/L) ${ }^{c}$

$0.20+1.00$

$0.21+2.41$

$0.36+0.94$

$0.20+0.39$

$0.18+1.21$

$0.17+0.63$

0.35

0.19-0.02

$0.52+1.31$

$0.24+1.68$

$0.13+6.13$

$0.26+2.34$

$0.20+0.41$

$0.14+0.94$

$0.15+0.94$

$0.29-0.40$

$0.17+1.46$

$0.21+1.43$

0.23

0.32

0.32

$0.23+2.79$

$0.18+2.21$

$0.20+0.37$

$0.19+0.67$

$0.23+0.30$

$0.26+0.91$

$0.27+0.40$

a $X^{\prime}=$ expected recovery for one or more measurements of a. sample containing a concentration of $C_{\text {, inmg/L. }}$.

b Expected single analyst standard deviation of measurements.

c Expected interlaboratory standard deviation of measurements.

d $C=$ true value for the concentration, in $\mathrm{mg} / \mathrm{L}$.

e $=$ average recovery found for measurements of samples containing a concentration of $C$, in $\mathrm{mg} / \mathrm{L}$.

$f$ Estimates based on performance of a single laboratory. 


\section{References}

EPA (U.S. Environmental Protection Agency), 1986. Test Methods for Evaluating Solid Waste (SW-846), Volumes IA-IC. Washington, DC.

EPA (U.S. Environmental Protection Agency), 1991. Guidelines Establishing Test Procedures for the Analysis of Pollutants, Code of Federal Regulations, Title 40, Part 136, Appendix A. Washington, DC.

EPA EMSL IU.S. Environmental Protection Agency, Environmental Monitoring and Systems Laboratory), 1980. Prescribed Procedures for Measurement of Radioactivity in Drinking Water, EPA-600/4-80-032. Cincinnati, $\mathrm{OH}$.

EPA EMSL (U.S. Environmental Protection Agency, Environmental Monitoring and Systems Laboratory), 1983. Methods for Chemical Analysis of Water and Wastes. Cincinnati, $\mathrm{OH}$.

EPA EMSL IU.S. Environmental Protection.Agency, Environmental Monitoring and Systems Laboratory); 1991. Test Method, The Determination of Inorganic Anions in Water by Ion Chromatography-Method 300.0. Cincinnati, $\mathrm{OH}$. 